\title{
Equivariant Perturbation in Gomory and Johnson's Infinite Group Problem. II. The Unimodular Two-Dimensional Case
}

\author{
Amitabh Basu, Robert Hildebrand, and Matthias Köppe \\ Dept. of Mathematics, University of California, Davis \\ abasu@math.ucdavis.edu, rhildebrand@math.ucdavis.edu, \\ mkoeppe@math.ucdavis.edu
}

\begin{abstract}
We give an algorithm for testing the extremality of a large class of minimal valid functions for the two-dimensional infinite group problem.
\end{abstract}

\section{Introduction}

\subsection{The group problem}

Gomory's group problem [8] is a central object in the study of strong cutting planes for integer linear optimization problems. One considers an abelian (not necessarily finite) group $G$, written additively, and studies the set of functions $s: G \rightarrow \mathbb{R}$ satisfying the following constraints:

$$
\begin{gathered}
\sum_{\mathbf{r} \in G} \mathbf{r} s(\mathbf{r}) \in \mathbf{f}+S \\
s(\mathbf{r}) \in \mathbb{Z}_{+} \text {for all } \mathbf{r} \in G \\
s \text { has finite support, }
\end{gathered}
$$

where $\mathbf{f}$ is a given element in $G$, and $S$ is a subgroup of $G$; so $\mathbf{f}+S$ is the coset containing the element $\mathbf{f}$. We will be concerned with the so-called infinite group problem [910], where $G=\mathbb{R}^{k}$ is taken to be the group of real $k$-vectors under addition, and $S=\mathbb{Z}^{k}$ is the subgroup of the integer vectors. We are interested in studying the convex hull $R_{\mathbf{f}}(G, S)$ of all functions satisfying the constraints in (IR). Observe that $R_{\mathbf{f}}(G, S)$ is a convex subset of the infinite-dimensional vector space $\mathcal{V}$ of functions $s: G \rightarrow \mathbb{R}$ with finite support.

Any linear inequality in $\mathcal{V}$ is given by a pair $(\pi, \alpha)$ where $\pi$ is a function $\pi: G \rightarrow \mathbb{R}$ (not necessarily of finite support) and $\alpha \in \mathbb{R}$. The linear inequality is then given by $\sum_{\mathbf{r} \in G} \pi(\mathbf{r}) s(\mathbf{r}) \geq \alpha$; the left-hand side is a finite sum because $s$ has finite support. Such an inequality is called a valid inequality for $R_{\mathbf{f}}(G, S)$ if $\sum_{\mathbf{r} \in G} \pi(\mathbf{r}) s(\mathbf{r}) \geq \alpha$ for all $s \in R_{\mathbf{f}}(G, S)$. It is customary to concentrate on those valid inequalities for which $\pi \geq 0$; then we can choose, after a scaling, $\alpha=1$. Thus, we only focus on valid inequalities of the form $\sum_{\mathbf{r} \in G} \pi(\mathbf{r}) s(\mathbf{r}) \geq 1$ with $\pi \geq 0$. Such functions $\pi$ will be termed valid functions for $R_{\mathbf{f}}(G, S)$. 
A valid function $\pi$ for $R_{\mathbf{f}}(G, S)$ is said to be minimal for $R_{\mathbf{f}}(G, S)$ if there is no valid function $\pi^{\prime} \neq \pi$ such that $\pi^{\prime}(\mathbf{r}) \leq \pi(\mathbf{r})$ for all $\mathbf{r} \in G$. For every valid function $\pi$ for $R_{\mathbf{f}}(G, S)$, there exists a minimal valid function $\pi^{\prime}$ such that $\pi^{\prime} \leq \pi$ (cf. [3]), and thus non-minimal valid functions are redundant in the description of $R_{\mathbf{f}}(G, S)$. Minimal functions for $R_{\mathbf{f}}(G, S)$ were characterized by Gomory for finite groups $G$ in $\left[8\right.$, and later for $R_{\mathbf{f}}(\mathbb{R}, \mathbb{Z})$ by Gomory and Johnson $[9]$. We state these results in a unified notation in the following theorem.

A function $\pi: G \rightarrow \mathbb{R}$ is subadditive if $\pi(\mathbf{x}+\mathbf{y}) \leq \pi(\mathbf{x})+\pi(\mathbf{y})$ for all $\mathbf{x}, \mathbf{y} \in G$. We say that $\pi$ is symmetric if $\pi(\mathbf{x})+\pi(\mathbf{f}-\mathbf{x})=1$ for all $\mathbf{x} \in G$.

Theorem 1.1 (Gomory and Johnson [9]). Let $\pi: G \rightarrow \mathbb{R}$ be a non-negative function. Then $\pi$ is a minimal valid function for $R_{\mathbf{f}}(G, S)$ if and only if $\pi(\mathbf{r})=0$ for all $\mathbf{r} \in S, \pi$ is subadditive, and $\pi$ satisfies the symmetry condition. (The first two conditions imply that $\pi$ is constant over any coset of $S$.)

\subsection{Characterization of extreme valid functions}

A stronger notion is that of an extreme function. A valid function $\pi$ is extreme for $R_{\mathbf{f}}(G, S)$ if it cannot be written as a convex combination of two other valid functions for $R_{\mathbf{f}}(G, S)$, i.e., $\pi=\frac{1}{2} \pi_{1}+\frac{1}{2} \pi_{2}$ implies $\pi=\pi_{1}=\pi_{2}$. Extreme functions are minimal. A tight characterization of extreme functions for $R_{\mathbf{f}}\left(\mathbb{R}^{k}, \mathbb{Z}^{k}\right)$ has eluded researchers for the past four decades now, however, various specific sufficient conditions for guaranteeing extremality 3/4/76|5|11] have been proposed. The standard technique for showing extremality is as follows. Suppose that $\pi=\frac{1}{2} \pi^{1}+\frac{1}{2} \pi^{2}$, where $\pi^{1}, \pi^{2}$ are other (minimal) valid functions. All subadditivity relations that are tight for $\pi$ are also tight for $\pi^{1}, \pi^{2}$. Then one uses a lemma of real analysis, the so-called Interval Lemma introduced by Gomory and Johnson in [11] or one of its variants. The Interval Lemma allows us to deduce certain affine linearity properties that $\pi^{1}$ and $\pi^{2}$ share with $\pi$. This is followed by a finite-dimensional linear algebra argument to establish uniqueness of $\pi$, implying $\pi=\pi^{1}=\pi^{2}$, and thus the extremality of $\pi$.

Surprisingly, the arithmetic (number-theoretic) aspect of the problem has been largely overlooked, even though it is at the core of the theory of the closely related finite group problem. In [2], the authors showed that this aspect is the key for completing the classification of extreme functions. The authors studied the case $k=1$ and gave a complete and algorithmic answer for the case of piecewise linear functions with rational breakpoints in the set $\frac{1}{q} \mathbb{Z}$. To capture the relevant arithmetics of the problem, the authors studied sets of additivity relations of the form $\pi\left(\mathbf{t}_{i}\right)+\pi(\mathbf{y})=\pi\left(\mathbf{t}_{i}+\mathbf{y}\right)$ and $\pi(\mathbf{x})+\pi\left(\mathbf{r}_{i}-\mathbf{x}\right)=\pi\left(\mathbf{r}_{i}\right)$, where the points $\mathbf{t}_{i}$ and $\mathbf{r}_{i}$ are certain breakpoints of the function $\pi$. They give rise to the reflection group $\Gamma$ generated by the reflections $\rho_{\mathbf{r}_{i}}: \mathbf{x} \mapsto \mathbf{r}_{i}-\mathbf{x}$ and translations $\tau_{\mathbf{t}_{i}}: \mathbf{y} \mapsto \mathbf{t}_{i}+\mathbf{y}$. The natural action of the reflection group $\Gamma$ on the set of intervals delimited by the elements of $\frac{1}{q} \mathbb{Z}$ transfers the affine linearity established by the Interval Lemma on some interval $I$ to a connected component of the orbit $\Gamma(I)$. When this establishes affine linearity of $\pi^{1}, \pi^{2}$ on all intervals where $\pi$ is affinely linear, one proceeds with finite-dimensional linear algebra 
to decide extremality of $\pi$. Otherwise, there is a way to perturb $\pi$ slightly to construct distinct minimal valid functions $\pi^{1}=\pi+\bar{\pi}$ and $\pi^{2}=\pi-\bar{\pi}$, using any sufficiently small, $\Gamma$-equivariant perturbation function (see subsection A.1), modified by restriction to a certain connected component.

\subsection{Contributions of the paper}

In the present paper, we continue the program of [2]. We study a remarkable class of minimal functions $\pi$ of the two-dimensional infinite group problem $(k=2)$. Let $q$ be a positive integer. Consider the arrangement $\mathcal{H}_{q}$ of all hyperplanes (lines) of the form $(0,1) \cdot \mathbf{x}=b,(1,0) \cdot \mathbf{x}=b$, and $(1,1) \cdot \mathbf{x}=b$, where $b \in \frac{1}{q} \mathbb{Z}$. The complement of the arrangement $\mathcal{H}_{q}$ consists of two-dimensional cells, whose closures are the triangles $T_{0}=\frac{1}{q} \operatorname{conv}\left(\left\{\left(\begin{array}{l}0 \\ 0\end{array}\right),\left(\begin{array}{l}1 \\ 0\end{array}\right),\left(\begin{array}{l}0 \\ 1\end{array}\right)\right\}\right)$ and $T_{1}=\frac{1}{q} \operatorname{conv}\left(\left\{\left(\begin{array}{l}1 \\ 0\end{array}\right),\left(\begin{array}{l}0 \\ 1\end{array}\right),\left(\begin{array}{l}1 \\ 1\end{array}\right)\right\}\right)$ and their translates by elements of the lattice $\frac{1}{q} \mathbb{Z}^{2}$. We denote by $\mathcal{P}_{q}$ the collection of these triangles and the vertices and edges that arise as intersections of the triangles. Thus $\mathcal{P}_{q}$ is a polyhedral complex that is a triangulation of the space $\mathbb{R}^{2}$. Within the polyhedral complex $\mathcal{P}_{q}$, let $\mathcal{P}_{q, 0}$ be the set of 0 -faces (vertices), $\mathcal{P}_{q, 1}$ be the set of 1 -faces (edges), and $\mathcal{P}_{q, 2}$ be the set of 2 -faces (triangles). The sets of diagonal, vertical, and horizontal edges will be denoted by $\mathcal{P}_{q, \backslash}, \mathcal{P}_{q, \mid}$, and $\mathcal{P}_{q,-}$, respectively. We will use $\oplus$ and $\ominus$ to denote vector addition and subtraction modulo 1 , respectively. We use the same notation for pointwise sums and differences of sets. By quotienting out by $\mathbb{Z}^{2}$, we obtain a finite complex that triangulates $\mathbb{R}^{2} / \mathbb{Z}^{2}$; we still denote it by $\mathcal{P}_{q}$.

We call a function $\pi: \mathbb{R}^{2} \rightarrow \mathbb{R}$ continuous piecewise linear over $\mathcal{P}_{q}$ if it is an affine linear function on each of the triangles of $\mathcal{P}_{q}$. We introduce the following notation. For every $I \in \mathcal{P}_{q}$, the restriction $\left.\pi\right|_{I}$ is an affine function, that is $\left.\pi\right|_{I}(\mathbf{x})=\mathbf{m}_{I} \cdot \mathbf{x}+b_{I}$ for some $\mathbf{m}_{I} \in \mathbb{R}^{2}, b_{I} \in \mathbb{R}$. We abbreviate $\left.\pi\right|_{I}$ as $\pi_{I}$.

For a valid function $\pi$, we consider the set $E(\pi)=\{(\mathbf{x}, \mathbf{y}) \mid \pi(\mathbf{x})+\pi(\mathbf{y})=$ $\pi(\mathbf{x} \oplus \mathbf{y})\}$ of pairs $(\mathbf{x}, \mathbf{y})$, for which the subadditivity relations are tight. Because $\mathcal{P}_{q}$ enjoys a strong unimodularity property (Lemma A.6), we can give a finite combinatorial representation of the set $E(\pi)$ using the faces of $\mathcal{P}_{q}$; this extends a technique in [2]. For faces $I, J, K \in \mathcal{P}_{q}$, let

$$
F(I, J, K)=\left\{(\mathbf{x}, \mathbf{y}) \in \mathbb{R}^{2} \times \mathbb{R}^{2} \mid \mathbf{x} \in I, \mathbf{y} \in J, \mathbf{x} \oplus \mathbf{y} \in K\right\} .
$$

A triple $(I, J, K)$ of faces is called a valid triple (Definition A.5) if none of the sets $I, J, K$ can individually decreased without changing the resulting set $F(I, J, K)$. Let $E\left(\pi, \mathcal{P}_{q}\right)$ denote the set of valid triples $(I, J, K)$ such that

$$
\pi(\mathbf{x})+\pi(\mathbf{y})=\pi(\mathbf{x} \oplus \mathbf{y}) \quad \text { for all } \quad(\mathbf{x}, \mathbf{y}) \in F(I, J, K) .
$$

$E\left(\pi, \mathcal{P}_{q}\right)$ is partially ordered by letting $(I, J, K) \leq\left(I^{\prime}, J^{\prime}, K^{\prime}\right)$ if and only if $I \subseteq I^{\prime}, J \subseteq J^{\prime}$, and $K \subseteq K^{\prime}$. Let $E_{\max }\left(\pi, \mathcal{P}_{q}\right)$ be the set of all maximal valid triples of the poset $E\left(\pi, \mathcal{P}_{q}\right)$. Then $E(\pi)$ is exactly covered by the sets $F(I, J, K)$ for the maximal valid triples $(I, J, K) \in E_{\max }\left(\pi, \mathcal{P}_{q}\right)$ (Lemma A.7).

In the present paper, we will restrict ourselves to a setting without maximal valid triples that include horizontal or vertical edges. 
Definition 1.2. A continuous piecewise linear function $\pi$ on $\mathcal{P}_{q}$ is called diagonally constrained if whenever $(I, J, K) \in E_{\max }\left(\pi, \mathcal{P}_{q}\right)$, then $I, J, K \in \mathcal{P}_{q, 0} \cup$ $\mathcal{P}_{q, \backslash} \cup \mathcal{P}_{q, 2}$.

Remark 1.3. Given a piecewise linear continuous valid function $\zeta: \mathbb{R} \rightarrow \mathbb{R}$ for the one-dimensional infinite group problem, Dey-Richard [5, Construction 6.1] consider the function $\kappa: \mathbb{R}^{2} \rightarrow \mathbb{R}, \kappa(\mathbf{x})=\zeta(\mathbf{1} \cdot \mathbf{x})$, where $\mathbf{1}=(1,1)$, and show that $\kappa$ is minimal and extreme if and only if $\zeta$ is minimal and extreme, respectively. If $\zeta$ has rational breakpoints in $\frac{1}{q} \mathbb{Z}$, then $\kappa$ belongs to our class of diagonally constrained continuous piecewise linear functions over $\mathcal{P}_{q}$.

We prove the following main theorem.

Theorem 1.4. Consider the following problem.

Given a minimal valid function $\pi$ for $R_{f}\left(\mathbb{R}^{2}, \mathbb{Z}^{2}\right)$ that is piecewise linear continuous on $\mathcal{P}_{q}$ and diagonally constrained, decide if $\pi$ is extreme.

There exists an algorithm for this problem that takes a number of elementary operations over the reals that is bounded by a polynomial in $q$.

As a direct corollary of the proof of the theorem, we obtain the following result relating the finite and infinite group problems.

Theorem 1.5. Let $\pi$ be a minimal continuous piecewise linear function over $\mathcal{P}_{q}$ that is diagonally constrained. Then $\pi$ is extreme for $R_{\mathbf{f}}\left(\mathbb{R}^{2}, \mathbb{Z}^{2}\right)$ if and only if the restriction $\left.\pi\right|_{\frac{1}{4 q} \mathbb{Z}^{2}}$ is extreme for $R_{\mathbf{f}}\left(\frac{1}{4 q} \mathbb{Z}^{2}, \mathbb{Z}^{2}\right)$.

We conjecture that the hypothesis on $\pi$ being diagonally constrained can be removed.

\section{Real analysis lemmas}

For any element $\mathbf{x} \in \mathbb{R}^{k}, k \geq 1,|\mathbf{x}|$ will denote the standard Euclidean norm. The proof of the following theorem appears in appendix A.3.

Theorem 2.1. If $\pi: \mathbb{R}^{k} \rightarrow \mathbb{R}$ is a minimal valid function, and $\pi=\frac{1}{2} \pi^{1}+$ $\frac{1}{2} \pi^{2}$, where $\pi^{1}, \pi^{2}$ are valid functions, then $\pi^{1}, \pi^{2}$ are both minimal. Moreover, if $\limsup _{\mathbf{h} \rightarrow 0} \frac{|\pi(\mathbf{h})|}{|\mathbf{h}|}<\infty$, then this condition also holds for $\pi^{1}$ and $\pi^{2}$. This implies that $\pi, \pi^{1}$ and $\pi^{2}$ are all Lipschitz continuous.

The following lemmas are corollaries of a general version of the interval lemma or similar real analysis arguments. Proofs appear in appendix A.7.

Lemma 2.2. Suppose $\pi$ is a continuous function and let $(I, J, K) \in E\left(\pi, \mathcal{P}_{q}\right)$ be a valid triple of triangles, i.e., $I, J, K \in \mathcal{P}_{q, 2}$. Then $\pi$ is affine in $I, J, K$ with the same gradient. 
Lemma 2.3. Suppose $\pi$ is a continuous function and let $(I, J, K) \in E\left(\pi, \mathcal{P}_{q}\right)$ where $I \in \mathcal{P}_{q, \backslash}, J, K \in \mathcal{P}_{q, \backslash} \cup \mathcal{P}_{q, 2}$. Then $\pi$ is affine in the diagonal direction in $I, J, K$, i.e., there exists $c \in \mathbb{R}$ such that such that $\pi\left(\mathbf{v}+\lambda\left(\begin{array}{c}-1 \\ 1\end{array}\right)\right)=\pi(\mathbf{v})+c \cdot \lambda$ for all $\mathbf{v} \in I$ (resp., $\mathbf{v} \in J, \mathbf{v} \in K$ ) and $\lambda \in \mathbb{R}$ such that $\mathbf{v}+\lambda\left(\begin{array}{c}-1 \\ 1\end{array}\right) \in I$ (resp., $\left.\mathbf{v}+\lambda\left(\begin{array}{c}-1 \\ 1\end{array}\right) \in J, \mathbf{v}+\lambda\left(\begin{array}{c}-1 \\ 1\end{array}\right) \in K\right)$.

Lemma 2.4. Let $I, J \in \mathcal{P}_{q, 2}$ be triangles such that $I \cap J \in \mathcal{P}_{q, \mid} \cup \mathcal{P}_{q,-}$. Let $\pi$ be a continuous function defined on $I \cup J$ satisfying the following properties:

(i) $\pi$ is affine on $I$.

(ii) There exists $c \in \mathbb{R}$ such that $\pi\left(\mathbf{v}+\lambda\left(\begin{array}{c}-1 \\ 1\end{array}\right)\right)=\pi(\mathbf{v})+c \cdot \lambda$ for all $\mathbf{v} \in J$ and $\lambda \in \mathbb{R}$ such that $\mathbf{v}+\lambda\left(\begin{array}{c}-1 \\ 1\end{array}\right) \in J$.

Then $\pi$ is affine on $J$.

\section{Proof of the main results}

Let $\partial_{\mathbf{v}}$ denote the directional derivative in the direction of $\mathbf{v}$.

Definition 3.1. Let $\pi$ be a minimal valid function.

(a) For any $I \in \mathcal{P}_{q}$, if $\pi$ is affine in $I$ and if for all valid functions $\pi^{1}, \pi^{2}$ such that $\pi=\frac{1}{2} \pi^{1}+\frac{1}{2} \pi^{2}$ we have that $\pi^{1}, \pi^{2}$ are affine in $I$, then we say that $\pi$ is affine imposing in $I$.

(b) For any $I \in \mathcal{P}_{q}$, if $\partial_{(-1,1)} \pi$ is constant in $I$ and if for all valid functions $\pi^{1}, \pi^{2}$ such that $\pi=\frac{1}{2} \pi^{1}+\frac{1}{2} \pi^{2}$ we have that $\partial_{(-1,1)} \pi^{1}, \partial_{(-1,1)} \pi^{2}$ are constant in $I$, then we say that $\pi$ is diagonally affine imposing in $I$.

(c) For a collection $\mathcal{P} \subseteq \mathcal{P}_{q}$, if for all $I \in \mathcal{P}, \pi$ is affine imposing (or diagonally affine imposing) in $I$, then we say that $\pi$ is affine imposing (diagonally affine imposing) in $\mathcal{P}$.

We either show that $\pi$ is affine imposing in $\mathcal{P}_{q}$ (subsection 3.1) or construct a continuous piecewise linear $\Gamma$-equivariant perturbation over $\mathcal{P}_{4 q}$ that proves $\pi$ is not extreme (subsections 3.2 and 3.3). If $\pi$ is affine imposing in $\mathcal{P}_{q}$, we set up a system of linear equations to decide if $\pi$ is extreme or not (subsection 3.4). This implies the main theorem stated in the introduction.

\subsection{Imposing affine linearity on faces of $\mathcal{P}_{q}$}

For the remainder of this paper, we will use reflections and translations modulo 1 to compensate for the fact that our function is periodic with period 1 . Working modulo 1 is accounted for by applying the translations $\tau_{(1,0)}$ and $\tau_{(0,1)}$ whenever needed. Hence, we define the reflection $\bar{\rho}_{\mathbf{v}}(\mathbf{x})=\mathbf{v} \ominus \mathbf{x}$ and the translation $\bar{\tau}_{\mathbf{v}}(\mathbf{x})=$ $\mathbf{v} \oplus \mathbf{x}$. The reflections and translations arise from certain valid triples as follows.

Lemma 3.2. Suppose $(I, J, K)$ is a valid triple. 
1. If $K=\{\mathbf{a}\} \in \mathcal{P}_{q, 0}$, then $J=\bar{\rho}_{\mathbf{a}}(I)$,

2. If $J=\{\mathbf{a}\} \in \mathcal{P}_{q, 0}$, then $K=\bar{\tau}_{\mathbf{a}}(I)$.

Proof. Part 1. Since $(I, J,\{\mathbf{a}\})$ is a valid triple, then for all $\mathbf{x} \in I$, there exists a $\mathbf{y} \in J$ such that $x \oplus \mathbf{y}=\mathbf{a}$, i.e., $\mathbf{y}=\mathbf{a} \ominus \mathbf{x} \in J$, and therefore $J \supseteq \bar{\rho}_{\mathbf{a}}(I)$. Also, for all $\mathbf{y} \in J$, there exists a $\mathbf{x} \in I$ such that $\mathbf{x} \oplus \mathbf{y}=\mathbf{a}$. Again, $\mathbf{y}=\mathbf{a} \ominus \mathbf{x}$, i.e., $J \subseteq \bar{\rho}_{\mathbf{a}}(I)$. Hence, $J=\bar{\rho}_{\mathbf{a}}(I)$.

Part 2. Since $(I,\{\mathbf{a}\}, K)$ is a valid triple and $J$ is a singleton, then for all $\mathbf{x} \in I$, we have $\mathbf{x} \oplus \mathbf{a} \in K$, i.e., $K \supseteq \bar{\tau}_{\mathbf{a}}(I)$. Also, for all $\mathbf{z} \in K$, there exists a $\mathbf{x} \in I$ such that $\mathbf{x} \oplus \mathbf{a}=\mathbf{z}$, i.e., $K \subseteq \bar{\tau}_{\mathbf{a}}(I)$. Hence, $K=\bar{\tau}_{\mathbf{a}}(I)$.

Let $\mathcal{G}=\mathcal{G}\left(\mathcal{P}_{q, 2}, \mathcal{E}\right)$ be an undirected graph with node set $\mathcal{P}_{q, 2}$ and edge set $\mathcal{E}=\mathcal{E}_{0} \cup \mathcal{E}$ 、 where $\{I, J\} \in \mathcal{E}_{0}\left(\{I, J\} \in \mathcal{E}_{\backslash}\right)$ if and only if for some $K \in \mathcal{P}_{q, 0}$ $\left(K \in \mathcal{P}_{q, \backslash}\right)$, we have $(I, J, K) \in E\left(\pi, \mathcal{P}_{q}\right)$ or $(I, K, J) \in E\left(\pi, \mathcal{P}_{q}\right)$. For each $I \in \mathcal{P}_{q, 2}$, let $\mathcal{G}_{I}$ be the connected component of $\mathcal{G}$ containing $I$.

We now consider faces of $\mathcal{P}_{q, 2}$ on which we will apply lemmas from section 2 .

$$
\begin{gathered}
\mathcal{P}_{q, 2}^{1}=\left\{I, J \in \mathcal{P}_{q, 2} \mid \exists K \in \mathcal{P}_{q, \backslash \text { with }}(I, J, K) \in E\left(\pi, \mathcal{P}_{q}\right) \text { or }(I, K, J) \in E\left(\pi, \mathcal{P}_{q}\right)\right\}, \\
\mathcal{P}_{q, 2}^{2}=\left\{I, J, K \in \mathcal{P}_{q, 2} \mid(I, J, K) \in E\left(\pi, \mathcal{P}_{q}\right)\right\} .
\end{gathered}
$$

It follows from Lemma 2.2 that $\pi$ is affine imposing in $\mathcal{P}_{q, 2}^{2}$ and from Lemma 2.3 that $\pi$ is diagonally affine imposing in $\mathcal{P}_{q, 2}^{1}$.

Faces connected in the graph have related slopes.

Lemma 3.3. Let $\mathbf{v} \in \mathbb{R}^{2}$. For $\theta=\pi, \pi^{1}$, or $\pi^{2}$, if $\theta$ is affine in the $\mathbf{v}$ direction in $I$, i.e., there exists $c \in \mathbb{R}$ such that such that $\pi(\mathbf{x}+\lambda \mathbf{v})=\pi(\mathbf{x})+c \cdot \lambda$ for all $\mathbf{x} \in I$ and $\lambda \in \mathbb{R}$ such that $\mathbf{x}+\lambda \mathbf{v} \in I$, and $\{I, J\} \in \mathcal{E}$, then $\theta$ is affine in the $\mathbf{v}$ direction in $J$ as well.

The proof appears in appendix A.8

With this in mind, we define the two sets of faces and any faces connected to them in the graph $\mathcal{G}$,

$$
\begin{aligned}
& \mathcal{S}_{q, 2}^{1}=\left\{J \in \mathcal{P}_{q, 2} \mid J \in \mathcal{G}_{I} \text { for some } I \in \mathcal{P}_{q, 2}^{1}\right\}, \\
& \mathcal{S}_{q, 2}^{2}=\left\{J \in \mathcal{P}_{q, 2} \mid J \in \mathcal{G}_{I} \text { for some } I \in \mathcal{P}_{q, 2}^{2}\right\} .
\end{aligned}
$$

It follows from Lemma 3.3 that $\pi$ is affine imposing in $\mathcal{S}_{q, 2}^{2}$ and diagonally affine imposing in $\mathcal{S}_{a, 2}^{1}$.

From Lemma 2.4 it follows that if $I \in \mathcal{S}_{q, 2}^{2}, J \in \mathcal{S}_{q, 2}^{1}$ and $I \cap J \in \mathcal{P}_{q, \mid} \cup \mathcal{P}_{q, \text {, }}$, then $\pi$ is affine imposing in $J$. Let

$\overline{\mathcal{S}}_{q, 2}=\left\{K \in \mathcal{G}_{I} \mid I \in \mathcal{S}_{q, 2}^{1}\right.$ and there exists a $J \in \mathcal{S}_{q, 2}^{2}$ such that $\left.I \cap J \in \mathcal{P}_{q, \mid} \cup \mathcal{P}_{q,-}\right\}$.

Now set $\overline{\mathcal{S}}_{q, 2}^{2}=\mathcal{S}_{q, 2}^{2} \cup \overline{\mathcal{S}}_{q, 2}$ and $\overline{\mathcal{S}}_{q, 2}^{1}=\mathcal{S}_{q, 2}^{1} \backslash \overline{\mathcal{S}}_{q, 2}$. The following theorem is a consequence of Lemmas 2.2, 2.4 , and 3.3 .

Theorem 3.4. If $\overline{\mathcal{S}}_{q, 2}^{2}=\mathcal{P}_{q, 2}$, then $\pi$ is affine imposing in $\mathcal{P}_{q, 2}$, and therefore $\theta$ is continuous piecewise linear over $\mathcal{P}_{q}$ for $\theta=\pi^{1}, \pi^{2}$. 


\subsection{Non-extremality by two-dimensional equivariant perturbation}

In this and the following subsection, we will prove the following result.

Lemma 3.5. Let $\pi$ be a minimal, continuous piecewise linear function over $\mathcal{P}_{q}$ that is diagonally constrained. If $\overline{\mathcal{S}}_{q, 2}^{2} \neq \mathcal{P}_{q, 2}$, then $\pi$ is not extreme.

In the proof, we will need two different equivariant perturbations that we construct as follows (see subsection A.1). Let $\Gamma_{0}=\left\langle\rho_{\mathbf{g}}, \tau_{\mathbf{g}} \mid \mathbf{g} \in \frac{1}{q} \mathbb{Z}^{2}\right\rangle$ be the group generated by reflections and translations corresponding to all possible vertices of $\mathcal{P}_{q}$. We define the function $\psi: \mathbb{R}^{2} \rightarrow \mathbb{R}$ as a continuous piecewise linear function over $\mathcal{P}_{4 q}$ in the following way: let $T_{0}=\frac{1}{q} \operatorname{conv}\left(\left\{\left(\begin{array}{l}0 \\ 0\end{array}\right),\left(\begin{array}{l}1 \\ 0\end{array}\right),\left(\begin{array}{l}0 \\ 1\end{array}\right)\right\}\right)$, and at all vertices of $\mathcal{P}_{4 q}$ that lie in $T_{0}$, let $\psi$ take the value 0 , except at the interior vertices $\frac{1}{4 q}\left(\begin{array}{l}1 \\ 1\end{array}\right), \frac{1}{4 q}\left(\begin{array}{l}2 \\ 1\end{array}\right), \frac{1}{4 q}\left(\begin{array}{l}1 \\ 2\end{array}\right)$, where we assign $\psi$ to have the value 1 . Since $\psi$ is continuous piecewise linear over $\mathcal{P}_{4 q}$, this uniquely determines the function on $T_{0}$. We then extend $\psi$ to all of $\mathbb{R}^{2}$ using the equivariance formula (2).

Lemma 3.6. The function $\psi: \mathbb{R}^{2} \rightarrow \mathbb{R}$ constructed above is well-defined and has the following properties:

(i) $\psi(\mathbf{g})=0$ for all $\mathbf{g} \in \frac{1}{q} \mathbb{Z}^{2}$,

(ii) $\psi(\mathbf{x})=-\psi\left(\rho_{\mathbf{g}}(\mathbf{x})\right)=-\psi(\mathbf{g}-\mathbf{x})$ for all $\mathbf{g} \in \frac{1}{q} \mathbb{Z}^{2}, \mathbf{x} \in[0,1]^{2}$,

(iii) $\psi(\mathbf{x})=\psi\left(\tau_{\mathbf{g}}(\mathbf{x})\right)=\psi(\mathbf{g}+\mathbf{x})$ for all $\mathbf{g} \in \frac{1}{q} \mathbb{Z}^{2}, \mathbf{x} \in[0,1]^{2}$,

(iv) $\psi$ is continuous piecewise linear over $\mathcal{P}_{4 q}$.

Proof. The properties follow directly from the equivariance formula (2).

It is now convenient to introduce the function $\Delta \pi(\mathbf{x}, \mathbf{y})=\pi(\mathbf{x})+\pi(\mathbf{y})-\pi(\mathbf{x} \oplus$ $\mathbf{y})$, which measures the slack in the subadditivity constraints. Let $\Delta \mathcal{P}_{q}$ be the polyhedral complex containing all polytopes $F=F(I, J, K)$ where $I, J, K \in \mathcal{P}_{q}$. Observe that $\left.\Delta \pi\right|_{F}$ is affine; if we introduce the function $\Delta \pi_{F}(\mathbf{x}, \mathbf{y})=\pi_{I}(\mathbf{x})+$ $\pi_{J}(\mathbf{y})-\pi_{K}(\mathbf{x} \oplus \mathbf{y})$ for all $\mathbf{x}, \mathbf{y} \in \mathbb{R}^{2}$, then $\Delta \pi(\mathbf{x}, \mathbf{y})=\Delta \pi_{F}(\mathbf{x}, \mathbf{y})$ for all $(\mathbf{x}, \mathbf{y}) \in F$. Furthermore, if $(I, J, K)$ is a valid triple, then $(I, J, K) \in E\left(\pi, \mathcal{P}_{q}\right)$ if and only if $\left.\Delta \pi\right|_{F(I, J, K)}=0$. We will use $\operatorname{vert}(F)$ to denote the set of vertices of the polytope $F$.

Lemma 3.7. Let $F \in \Delta \mathcal{P}_{q}$ and let $(\mathbf{x}, \mathbf{y})$ be a vertex of $F$. Then $\mathbf{x}, \mathbf{y}$ are vertices of the complex $\mathcal{P}_{q}$, i.e., $\mathbf{x}, \mathbf{y} \in \frac{1}{q} \mathbb{Z}^{2}$.

The proof again uses the strong unimodularity properties of $\mathcal{P}_{q}$ and appears in appendix A.2.

Lemma 3.8. Let $\pi$ be a minimal, continuous piecewise linear function over $\mathcal{P}_{q}$ that is diagonally constrained. Suppose there exists $I^{*} \in \mathcal{P}_{q, 2} \backslash\left(\overline{\mathcal{S}}_{q, 2}^{2} \cup \overline{\mathcal{S}}_{q, 2}^{1}\right)$. Then $\pi$ is not extreme.

Proof. Let $R=\bigcup_{J \in \mathcal{G}_{I^{*}}} \operatorname{int}(J) \subseteq[0,1]^{2}$. Since $R$ is a union of interiors, it does not contain any points in $\frac{1}{2 q} \mathbb{Z}^{2}$. Let $\psi$ be the $\Gamma_{0}$-equivariant function of Lemma 3.6 . Let

$$
\epsilon=\min \left\{\Delta \pi_{\hat{F}}(\mathbf{x}, \mathbf{y}) \neq 0 \mid \hat{F} \in \Delta \mathcal{P}_{4 q},(\mathbf{x}, \mathbf{y}) \in \operatorname{vert}(\hat{F})\right\},
$$


and let $\bar{\pi}=\delta_{R} \cdot \psi$ where $\delta_{R}$ is the indicator function for the set $R$. We will show that for

$$
\pi^{1}=\pi+\frac{\epsilon}{3} \bar{\pi}, \quad \pi^{2}=\pi-\frac{\epsilon}{3} \bar{\pi},
$$

that $\pi^{1}, \pi^{2}$ are minimal, and therefore valid functions, and hence $\pi$ is not extreme. We will show this just for $\pi^{1}$ as the proof for $\pi^{2}$ is the same.

Since $\psi(\mathbf{0})=0$ and $\psi(\mathbf{f})=0$, we see that $\pi^{1}(\mathbf{0})=0$ and $\pi^{1}(\mathbf{f})=1$.

We want to show that $\pi^{1}$ is symmetric and subadditive. We will do this by analyzing the function $\Delta \pi^{1}(\mathbf{x}, \mathbf{y})=\pi^{1}(\mathbf{x})+\pi^{1}(\mathbf{y})-\pi^{1}(\mathbf{x} \oplus \mathbf{y})$. Since $\psi$ is piecewise linear over $\mathcal{P}_{4 q}, \pi^{1}$ is also piecewise linear over $\mathcal{P}_{4 q}$, and thus we only need to focus on vertices of $\Delta \mathcal{P}_{4 q}$, which, by Lemma 3.7 , are contained in $\frac{1}{4 q} \mathbb{Z}^{2}$.

Let $\mathbf{u}, \mathbf{v} \in \frac{1}{4 q} \mathbb{Z}^{2}$. First, if $\left.\Delta \pi_{(} \mathbf{u}, \mathbf{v}\right)>0$, then

$$
\Delta \pi^{1}(\mathbf{u}, \mathbf{v}) \geq \pi(\mathbf{u})-\epsilon / 3+\pi(\mathbf{v})-\epsilon / 3-\pi(\mathbf{u} \oplus \mathbf{v})-\epsilon / 3=\Delta \pi(\mathbf{u}, \mathbf{v})-\epsilon \geq 0 .
$$

Next, we will show that if $\Delta \pi(\mathbf{u}, \mathbf{v})=0$, then $\Delta \pi^{1}(\mathbf{u}, \mathbf{v})=0$. This will prove two things. First, $\Delta \pi^{1}(\mathbf{x}, \mathbf{y}) \geq 0$ for all $\mathbf{x}, \mathbf{y} \in[0,1]^{2}$, and therefore $\pi^{1}$ is subadditive. Second, since $\pi$ is symmetric, $\Delta \pi(\mathbf{x}, \mathbf{f} \ominus \mathbf{x})=0$ for all $\mathbf{x} \in \frac{1}{4 q} \mathbb{Z}^{2}$, which would imply that $\Delta \pi^{1}(\mathbf{x}, \mathbf{f} \ominus \mathbf{x})=0$ for all $\mathbf{x} \in \frac{1}{4 q} \mathbb{Z}^{2}$, proving $\pi^{1}$ is symmetric via Lemma A.9.

Suppose that $\Delta \pi(\mathbf{u}, \mathbf{v})=0$. We will proceed by cases.

Case 1. Suppose $\mathbf{u}, \mathbf{v}, \mathbf{u} \oplus \mathbf{v} \notin R$. Then $\delta_{R}(\mathbf{u})=\delta_{R}(\mathbf{v})=\delta_{R}(\mathbf{u} \oplus \mathbf{v})=0$, and $\Delta \pi^{1}(\mathbf{u}, \mathbf{v})=\Delta \pi(\mathbf{u}, \mathbf{v}) \geq 0$.

Case 2. Suppose we are not in Cases 1 . That is, suppose $\Delta \pi(\mathbf{u}, \mathbf{v})=0$, and at least one of $\mathbf{u}, \mathbf{v}, \mathbf{u} \oplus \mathbf{v}$ is in $R$. Since $R \cap \frac{1}{2 q} \mathbb{Z}^{2}=\emptyset$, at least one of $\mathbf{u}, \mathbf{v} \mathbf{u} \oplus \mathbf{v} \notin \frac{1}{2 q} \mathbb{Z}^{2}$. This implies that at least one of $\mathbf{u}, \mathbf{v} \notin \frac{1}{2 q} \mathbb{Z}^{2}$. Since $\Delta \pi^{1}(\mathbf{x}, \mathbf{y})$ is symmetric in $\mathbf{x}$ and $\mathbf{y}$, without loss of generality, we will assume that $\mathbf{u} \notin \frac{1}{2 q} \mathbb{Z}^{2}$.

Since $\mathbf{u} \notin \frac{1}{2 q} \mathbb{Z}^{2},(\mathbf{u}, \mathbf{v}) \notin \operatorname{vert}\left(\Delta \mathcal{P}_{q}\right)$. Therefore, there exists a face $F \in$ $\Delta \mathcal{P}_{q}$ such that $(\mathbf{u}, \mathbf{v}) \in \operatorname{rel} \operatorname{int}(F)$. Since $\Delta \pi_{F} \geq 0(\pi$ is subadditive $)$ and $\Delta \pi_{F}(\mathbf{u}, \mathbf{v})=0$, it follows that $\Delta \pi_{F}=0$. Now let $(I, J, K) \in E_{\max }\left(\pi, \mathcal{P}_{q}\right)$ such that $F(I, J, K) \supseteq F$. We discuss the possible cases for $I, J, K$ from Lemma A.11.

1. If $I, J, K \notin \mathcal{P}_{q, 2}$, then $I, J, K \in \mathcal{P}_{q, 0} \cup \mathcal{P}_{q}$, are all vertices or edges of $\mathcal{P}_{q}$, which are all not contained in $R$ since $R$ is the union of interiors of sets from $\mathcal{P}_{q, 2}$. Therefore, $\mathbf{u}, \mathbf{v}, \mathbf{u} \oplus \mathbf{v} \notin R$, which means we are in Case 1 .

2. If $I, J, K \in \mathcal{P}_{q, 2}$, then $I, J, K \in \mathcal{S}_{q, 2}^{2}$. Therefore, $\mathbf{u}, \mathbf{v}, \mathbf{u} \oplus \mathbf{v} \notin R$, which means we are in Case 1.

3. One of $I, J, K$ is a diagonal edge in $\mathcal{P}_{q, 1}$, while the other two are in $\mathcal{P}_{q, 2}$, which means these sets are in $\mathcal{S}_{q, 2}^{1}$. Since edges are not in $R$, and $R \cap \mathcal{S}_{q, 2}^{1}=\emptyset$, and again, $\mathbf{u}, \mathbf{v}, \mathbf{u} \oplus \mathbf{v} \notin R$, which means we are in Case 1 .

4. This leaves us with the case where two of $I, J, K$ are in $\mathcal{P}_{q, 2}$ and the third is a vertex, i.e., is in $\mathcal{P}_{q, 0}$. Since $\mathbf{u} \notin \frac{1}{q} \mathbb{Z}^{2}, I$ cannot be a vertex. Therefore, $I \in \mathcal{P}_{q, 2}$. We proceed with this knowledge.

There are two possible cases.

Case 2a. $J \in \mathcal{P}_{q, 0}, I, K \in \mathcal{P}_{q, 2}$ and hence $\mathbf{v} \in \frac{1}{q} \mathbb{Z}^{2}$. 
Therefore $\{I, K\} \in \mathcal{E}_{0}$ and $\delta_{R}(\mathbf{u})=\delta_{R}(\mathbf{u} \oplus \mathbf{v})$. Since $\mathbf{v} \in \frac{1}{q} \mathbb{Z}^{2}$, we have $\psi(\mathbf{v})=0$ and $\psi(\mathbf{u})=\psi\left(\bar{\tau}_{\mathbf{v}}(\mathbf{u})\right)=\psi(\mathbf{u} \oplus \mathbf{v})$ by Lemma 3.6 (iii). It follows that $\bar{\pi}(\mathbf{u})+$ $\bar{\pi}(\mathbf{v})-\bar{\pi}(\mathbf{u} \oplus \mathbf{v})=0$, and therefore $\Delta \pi^{1}(\mathbf{u}, \mathbf{v})=\Delta \pi(\mathbf{u}, \mathbf{v})=0$.

Case 2b. $I, J \in \mathcal{P}_{q, 2}, K \in \mathcal{P}_{q, 0}$ and hence $\mathbf{u} \oplus \mathbf{v} \in \frac{1}{q} \mathbb{Z}^{2}$. Therefore $\{I, J\} \in \mathcal{E}_{0}$ and $\delta_{R}(\mathbf{u})=\delta_{R}(\mathbf{v})$. Since $\mathbf{u} \oplus \mathbf{v} \in \frac{1}{q} \mathbb{Z}^{2}, \psi(\mathbf{u})=-\psi\left(\bar{\rho}_{\mathbf{u} \oplus \mathbf{v}}(\mathbf{u})\right)=-\psi(\mathbf{v})$ by Lemma 3.6 (ii). It follows that $\bar{\pi}(\mathbf{u})+\bar{\pi}(\mathbf{v})-\bar{\pi}(\mathbf{u} \oplus \mathbf{v})=0$, and therefore $\Delta \pi^{1}(\mathbf{u}, \mathbf{v})=\Delta \pi(\mathbf{u}, \mathbf{v})=0$.

We conclude that $\pi^{1}$ (and similarly $\pi^{2}$ ) is subadditive and symmetric, and therefore minimal and hence valid. Therefore $\pi$ is not extreme.

\subsection{Non-extremality by diagonal equivariant perturbation}

We next construct a different equivariant perturbation function. Let $\Gamma=$ $\left\langle\rho_{\mathbf{g}}, \tau_{\mathbf{g}} \mid \mathbf{1} \cdot \mathbf{g} \equiv 0\left(\bmod \frac{1}{q}\right)\right\rangle$, where $\mathbf{1}=(1,1)$, be the group generated by reflections and translations corresponding to all points on diagonal edges of $\mathcal{P}_{q}$. We define the function $\varphi: \mathbb{R}^{2} \rightarrow \mathbb{R}$ as a continuous piecewise linear function over $\mathcal{P}_{4 q}$ in the following way:

$$
\varphi(\mathbf{x})= \begin{cases}1 & \text { if } \mathbf{1} \cdot \mathbf{x} \equiv \frac{1}{4 q} \quad\left(\bmod \frac{1}{q}\right) \\ -1 & \text { if } \mathbf{1} \cdot \mathbf{x} \equiv \frac{3}{4 q} \quad\left(\bmod \frac{1}{q}\right) \\ 0 & \text { if } \mathbf{1} \cdot \mathbf{x} \equiv 0 \text { or } \frac{2}{4 q} \quad\left(\bmod \frac{1}{q}\right) .\end{cases}
$$

This function satisfies all properties of Lemma 3.6 but is also $\Gamma_{\backslash}$-equivariant.

Lemma 3.9. Suppose there exists $I^{*} \in \overline{\mathcal{S}}_{q, 2}^{1}$ and $\pi$ is diagonally constrained. Then $\pi$ is not extreme.

Proof. Let $R=\left(\bigcup_{J \in \mathcal{G}_{I^{*}}} J\right) \backslash\left\{\mathbf{x} \mid \mathbf{1} \cdot \mathbf{x} \equiv 0\right.$ or $\left.\frac{2}{4 q}\left(\bmod \frac{1}{q}\right)\right\}$.

Let

$$
\epsilon=\min \left\{\Delta \pi_{F}(\mathbf{x}, \mathbf{y}) \neq 0 \mid F \in \Delta \mathcal{P}_{4 q},(\mathbf{x}, \mathbf{y}) \in \operatorname{vert}(F)\right\},
$$

and let $\bar{\pi}$ be the unique continuous piecewise linear function over $\mathcal{P}_{4 q}$ such that for any vertex $\mathbf{x}$ of $\mathcal{P}_{4 q}$, we have $\bar{\pi}(\mathbf{x})=\delta_{R}(\mathbf{x}) \cdot \varphi(\mathbf{x})$ where $\delta_{R}$ is the indicator function for the set $R$. By construction, $\bar{\pi}$ is a continuous function that vanishes on all diagonal hyperplanes in the complex $\mathcal{P}_{q}$. We will show that for

$$
\pi^{1}=\pi+\frac{\epsilon}{3} \bar{\pi}, \quad \pi^{2}=\pi-\frac{\epsilon}{3} \bar{\pi},
$$

that $\pi^{1}, \pi^{2}$ are minimal, and therefore valid functions, and hence $\pi$ is not extreme. We will show this just for $\pi^{1}$ as the proof for $\pi^{2}$ is the same.

Since, $\varphi(\mathbf{0})=0$ and $\varphi(\mathbf{f})=0$, we see that $\pi^{1}(\mathbf{0})=0$ and $\pi^{1}(\mathbf{f})=1$.

We want to show that $\pi^{1}$ is symmetric and subadditive. We will do this by analyzing the function $\Delta \pi^{1}(\mathbf{x}, \mathbf{y})=\pi^{1}(\mathbf{x})+\pi^{1}(\mathbf{y})-\pi^{1}(\mathbf{x} \oplus \mathbf{y})$. Since $\bar{\pi}$ is continuous piecewise linear over $\mathcal{P}_{4 q}, \pi^{1}$ is also continuous piecewise linear over 
$\mathcal{P}_{4 q}$, and thus we only need to focus on vertices of $\Delta \mathcal{P}_{4 q}$, which, by Lemma 3.7 , are contained in $\frac{1}{4 q} \mathbb{Z}^{2}$.

Let $\mathbf{u}, \mathbf{v} \in \frac{1}{4 q} \mathbb{Z}^{2}$.

First, if $\left.\Delta \pi_{(} \mathbf{u}, \mathbf{v}\right)>0$, then $\left.\Delta \pi_{(} \mathbf{u}, \mathbf{v}\right) \geq \epsilon$ and therefore

$$
\Delta \pi^{1}(\mathbf{u}, \mathbf{v}) \geq \pi(\mathbf{u})-\epsilon / 3+\pi(\mathbf{v})-\epsilon / 3-\pi(\mathbf{u} \oplus \mathbf{v})-\epsilon / 3=\Delta \pi(\mathbf{u}, \mathbf{v})-\epsilon \geq 0
$$

Next, we will show that if $\Delta \pi(\mathbf{u}, \mathbf{v})=0$, then $\Delta \pi^{1}(\mathbf{u}, \mathbf{v})=0$. This will prove two things. First, $\Delta \pi^{1}(\mathbf{x}, \mathbf{y}) \geq 0$ for all $\mathbf{x}, \mathbf{y} \in[0,1]^{2}$, and therefore $\pi^{1}$ is subadditive. Second, since $\pi$ is symmetric, $\Delta \pi(\mathbf{x}, \mathbf{f} \ominus \mathbf{x})=0$ for all $\mathbf{x} \in \frac{1}{4 q} \mathbb{Z}^{2}$, which would imply that $\Delta \pi^{1}(\mathbf{x}, \mathbf{f} \ominus \mathbf{x})=0$ for all $\mathbf{x} \in \frac{1}{4 q} \mathbb{Z}^{2}$, proving $\pi^{1}$ is symmetric via Lemma A.9.

Suppose that $\Delta \pi(\mathbf{u}, \mathbf{v})=0$. We will proceed by cases.

Case 1. Suppose $\mathbf{u}, \mathbf{v}, \mathbf{u} \oplus \mathbf{v} \notin R$. Then $\delta_{R}(\mathbf{u})=\delta_{R}(\mathbf{v})=\delta_{R}(\mathbf{u} \oplus \mathbf{v})=0$, and $\Delta \pi^{1}(\mathbf{u}, \mathbf{v})=\Delta \pi(\mathbf{u}, \mathbf{v}) \geq 0$.

Case 2. Suppose $\mathbf{u}, \mathbf{v} \in \frac{1}{2 q} \mathbb{Z}^{2}$. Then $\mathbf{1} \cdot(\mathbf{u} \oplus \mathbf{v}) \equiv 0\left(\bmod \frac{1}{q}\right)$ and, by definition of $R, \mathbf{u}, \mathbf{v}, \mathbf{u} \oplus \mathbf{v} \notin R$, and we are actually in Case 1 .

Case 3. Suppose we are not in Cases 1 or 2. That is, suppose $\Delta \pi(\mathbf{u}, \mathbf{v})=0$, not both $\mathbf{u}, \mathbf{v}$ are in $\frac{1}{2 q} \mathbb{Z}^{2}$, and at least one of $\mathbf{u}, \mathbf{v}, \mathbf{u} \oplus \mathbf{v}$ is in $R$. Since $\Delta \pi^{1}(\mathbf{x}, \mathbf{y})$ is symmetric in $\mathbf{x}$ and $\mathbf{y}$, without loss of generality, since not both $\mathbf{u}, \mathbf{v}$ are in $\frac{1}{2 q} \mathbb{Z}^{2}$, we will assume that $\mathbf{u} \notin \frac{1}{2 q} \mathbb{Z}^{2}$.

Since $\mathbf{u} \notin \frac{1}{2 q} \mathbb{Z}^{2},(\mathbf{u}, \mathbf{v}) \notin \operatorname{vert}\left(\Delta \mathcal{P}_{q}\right)$. Therefore, there exists a face $F \in$ $\Delta \mathcal{P}_{q}$ such that $(\mathbf{u}, \mathbf{v}) \in \operatorname{rel} \operatorname{int}(F)$. Since $\Delta \pi_{F} \geq 0(\pi$ is subadditive $)$ and $\Delta \pi_{F}(\mathbf{u}, \mathbf{v})=0$, it follows that $\Delta \pi_{F}=0$. Now let $(I, J, K) \in E_{\max }\left(\pi, \mathcal{P}_{q}\right)$ such that $F(I, J, K) \supseteq F$. Since $\pi$ is diagonally constrained, by definition, $I, J, K$ are each either a vertex, diagonal edge, or triangle in $\mathcal{P}_{q}$. We discuss the possible cases for $I, J, K$ according to Lemma A.11.

1. If $I, J, K \notin \mathcal{P}_{q, 2}$, then $I, J, K$ are all vertices or diagonal edges of $\mathcal{P}_{q}$, which are all not contained in $R$ since all vertices and diagonal edges are subsets of $\left\{\mathbf{x} \mid \mathbf{x}_{1}+\mathbf{x}_{2} \equiv 0\left(\bmod \frac{1}{q}\right)\right\}$. Therefore, $\mathbf{u}, \mathbf{v}, \mathbf{u} \oplus \mathbf{v} \notin R$, which means we are in Case 1.

2. If $I, J, K \in \mathcal{P}_{q, 2}$, then $I, J, K \in \mathcal{S}_{q, 2}^{2}$. By definition of $\overline{\mathcal{S}}_{q, 2}^{1}$, for any $I^{\prime} \in \mathcal{S}_{q, 2}^{2}$ and $J^{\prime} \in \overline{\mathcal{S}}_{q, 2}^{1}$, either $I^{\prime} \cap J^{\prime}=\emptyset$, or $I^{\prime} \cap J^{\prime} \in \mathcal{P}_{q, \backslash}$. Therefore, $\mathbf{u}, \mathbf{v}, \mathbf{u} \oplus \mathbf{v} \notin R$, which means we are in Case 1 .

3. If two of $I, J, K$ are in $\mathcal{P}_{q, 2}$ and the third is a vertex, i.e., is in $\mathcal{P}_{q, 0}$. Since $\mathbf{u} \notin \frac{1}{q} \mathbb{Z}^{2}, I$ cannot be a vertex. Therefore, $I \in \mathcal{P}_{q, 2}$. For this case, the proof is exactly the same as Case $2 \mathrm{a}$ and Case $2 \mathrm{~b}$ in the proof of Lemma 3.8 because $\bar{\pi}(\mathbf{x})=0$ for all vertices $\mathbf{x} \in \mathcal{P}_{q, 0}$. For brevity, we will not repeat it here.

4. If one of $I, J, K$ is in $\mathcal{P}_{q, \backslash}$, call it $I^{\prime}$, and the other two are in $\mathcal{P}_{q, 2}$, call them $J^{\prime}, K^{\prime}$, then $J^{\prime}, K^{\prime} \in \mathcal{S}_{q, 2}^{1}$ and $\left\{J^{\prime}, K^{\prime}\right\} \in \mathcal{E} \backslash$. Since $I^{\prime} \in \mathcal{P}_{q, \backslash,}, I^{\prime} \cap R=\emptyset$. Recall that $\mathcal{S}_{q, 2}^{1} \subseteq \overline{\mathcal{S}}_{q, 2}^{1} \cup \overline{\mathcal{S}}_{q, 2}^{2}$. If either $J^{\prime}$ or $K^{\prime}$ is in $\overline{\mathcal{S}}_{q, 2}^{2}$, then they both are in $\overline{\mathcal{S}}_{q, 2}^{2}$, i.e., $J^{\prime} \cup K^{\prime} \cap R=\emptyset$ and therefore $\mathbf{u}, \mathbf{v}, \mathbf{u} \oplus \mathbf{v} \notin R$, which is Case 1 . We proceed to consider the case where $I^{\prime} \in \mathcal{P}_{q, \backslash}$ and $J^{\prime}, K^{\prime} \in \overline{\mathcal{S}}_{q, 2}^{1}$ with $\left\{J^{\prime}, K^{\prime}\right\} \in \mathcal{E} \backslash$ of which there are three possible cases. 
Case 3a. $I \in \mathcal{P}_{q, \backslash}, J, K \in \mathcal{P}_{q, 2}$. Since $\{J, K\} \in \mathcal{E} \backslash, \delta_{R}(\mathbf{v})=\delta_{R}(\mathbf{u} \oplus \mathbf{v})$. Since $I \in \mathcal{P}_{q, \backslash}$ and $\mathbf{u} \in I, \mathbf{1} \cdot \mathbf{u} \equiv 0\left(\bmod \frac{1}{q}\right)$. It follows that $\varphi(\mathbf{u})=0$ and $\mathbf{1} \cdot \mathbf{v} \equiv \mathbf{1} \cdot(\mathbf{u} \oplus \mathbf{v})\left(\bmod \frac{1}{q}\right)$. Therefore, $\varphi(\mathbf{v})=\varphi(\mathbf{u} \oplus \mathbf{v})$. Combining these, we have $\bar{\pi}(\mathbf{u})+\bar{\pi}(\mathbf{v})-\bar{\pi}(\mathbf{u} \oplus \mathbf{v})=0$, and therefore $\Delta \pi^{1}(\mathbf{u}, \mathbf{v})=\Delta \pi(\mathbf{u}, \mathbf{v})=0$.

Case 3b. $J \in \mathcal{P}_{q, \backslash,}, K, K \in \mathcal{P}_{q, 2}$. This is similar to Case 3a and the proof need not be repeated.

Case 3c. $I, J \in \mathcal{P}_{q, 2}, K \in \mathcal{P}_{q, \backslash}$ and hence $\mathbf{1} \cdot(\mathbf{u} \oplus \mathbf{v}) \equiv 0\left(\bmod \frac{1}{q}\right)$. Since $\{I, J\} \in \mathcal{E}$, we have $\delta_{R}(\mathbf{u})=\delta_{R}(\mathbf{v})$. Since $\mathbf{1} \cdot(\mathbf{u} \oplus \mathbf{v}) \equiv 0\left(\bmod \frac{1}{q}\right)$, we have $\mathbf{1} \cdot \mathbf{u} \equiv-\mathbf{1} \cdot \mathbf{v}\left(\bmod \frac{1}{q}\right)$, and hence $\varphi(\mathbf{u})=-\varphi(\mathbf{v})$. It follows that $\bar{\pi}(\mathbf{u})+\bar{\pi}(\mathbf{v})-$ $\bar{\pi}(\mathbf{u} \oplus \mathbf{v})=0$, and therefore $\Delta \pi^{1}(\mathbf{u}, \mathbf{v})=\Delta \pi(\mathbf{u}, \mathbf{v})=0$.

We conclude that $\pi^{1}$ (and similarly $\pi^{2}$ ) is subadditive and symmetric, and therefore minimal and hence valid. Therefore $\pi$ is not extreme.

Proof (of Lemma 3.5). This follows directly from Lemmas 3.8 and 3.9

The specific form of our perturbations as continuous piecewise linear function over $\mathcal{P}_{4 q}$ implies the following corollary.

Corollary 3.10. Suppose $\pi$ is a continuous piecewise linear function over $\mathcal{P}_{q}$ and is diagonally constrained. If $\pi$ is not affine imposing over $\mathcal{P}_{q, 2}$, then there exist distinct minimal $\pi^{1}, \pi^{2}$ that are continuous piecewise linear over $\mathcal{P}_{4 q}$ such that $\pi=\frac{1}{2} \pi^{1}+\frac{1}{2} \pi^{2}$.

\subsection{Extremality and non-extremality by linear algebra}

In this section we suppose $\pi$ is a minimal continuous piecewise linear function over $\mathcal{P}_{q}$ that is affine imposing in $\mathcal{P}_{q, 2}$. Therefore, $\pi^{1}$ and $\pi^{2}$ must also be continuous piecewise linear functions over $\mathcal{P}_{q}$. It is clear that whenever $\pi(\mathbf{x})+\pi(\mathbf{y})=$ $\pi(\mathbf{x} \oplus \mathbf{y})$, the functions $\pi^{1}$ and $\pi^{2}$ must also satisfy this equality relation, that is, $\pi^{i}(\mathbf{x})+\pi^{i}(\mathbf{y})=\pi^{i}(\mathbf{x} \oplus \mathbf{y})$. We now set up a system of linear equations that $\pi$ satisfies and that $\pi_{1}$ and $\pi_{2}$ must also satisfy. Let $\varphi: \frac{1}{q} \mathbb{Z}^{2} \rightarrow \mathbb{R}$. Suppose $\varphi$ satisfies the following system of linear equations:

$$
\left\{\begin{array}{l}
\left.\varphi(\mathbf{0})=0, \varphi(\mathbf{f})=1, \varphi\left(\left(\begin{array}{l}
0 \\
1
\end{array}\right)\right)=0, \varphi\left(\left(\begin{array}{l}
0 \\
1
\end{array}\right)\right)=0, \varphi\left(\left(\begin{array}{l}
1 \\
1
\end{array}\right)\right)\right)=0, \\
\varphi(\mathbf{u})+\varphi(\mathbf{v})=\varphi(\mathbf{u} \oplus \mathbf{v}) \text { if } \mathbf{u}, \mathbf{v} \in \frac{1}{q} \mathbb{Z}^{2}, \pi(\mathbf{u})+\pi(\mathbf{v})=\pi(\mathbf{u} \oplus \mathbf{v})
\end{array}\right.
$$

Since $\pi$ exists and satisfies (1), we know that the system has a solution.

Theorem 3.11. Let $\pi: \mathbb{R}^{2} \rightarrow \mathbb{R}$ be a continuous piecewise linear valid function over $\mathcal{P}_{q}$.

i. If the system (1) does not have a unique solution, then $\pi$ is not extreme.

ii. Suppose $\pi$ is minimal and affine imposing in $\mathcal{P}_{q, 2}$. Then $\pi$ is extreme if and only if the system of equations (1) has a unique solution.

The proof, similar to one in 2], appears in appendix A.9. 


\subsection{Connection to a finite group problem}

Theorem 3.12. Let $\pi$ be a minimal continuous piecewise linear function over $\mathcal{P}_{q}$ that is diagonally constrained. Then $\pi$ is extreme if and only if the system of equations (1) with $\frac{1}{4 q} \mathbb{Z}^{2}$ has a unique solution.

Proof. Since $\pi$ is continuous piecewise linear over $\mathcal{P}_{q}$, it is also continuous piecewise linear over $\mathcal{P}_{4 q}$. The forward direction is the contrapositive of Theorem 3.11(i), applied when we view $\pi$ piecewise linear over $\mathcal{P}_{4 q}$. For the reverse direction, observe that if the system of equations (1) with $\frac{1}{4 q} \mathbb{Z}^{2}$ has a unique solution, then there cannot exist distinct minimal $\pi^{1}, \pi^{2}$ that are continuous piecewise linear over $\mathcal{P}_{4 q}$ such that $\pi=\frac{1}{2} \pi^{1}+\frac{1}{2} \pi^{2}$. By the contrapositive of Corollary $3.10 \pi$ is affine imposing in $\mathcal{P}_{q, 2}$. Then $\pi$ is also affine imposing on $\mathcal{P}_{4 q, 2}$ since it is a finer set. By Theorem 3.11(ii), since $\pi$ is affine imposing in $\mathcal{P}_{4 q, 2}$ and the system of equations (1) on $\mathcal{P}_{4 q}$ has a unique solution, $\pi$ is extreme.

Theorem 1.4 is proved by testing for minimality using Lemma A.10 and then testing for extremality using Theorem 3.12 . Theorem 1.5 is a direct consequence of Theorem 3.12 ,

\section{References}

1. Amitabh Basu, Michele Conforti, Gérard Cornuéjols, and Giacomo Zambelli. A counterexample to a conjecture of Gomory and Johnson. Mathematical Programming Ser. A, 133:25-38, 2012.

2. Amitabh Basu, Robert Hildebrand, and Matthias Köppe. Equivariant perturbation in Gomory and Johnson's infinite group problem. eprint arXiv:1206.2079 [math.OC], 2012. manuscript.

3. Amitabh Basu, Robert Hildebrand, Matthias Köppe, and Marco Molinaro. A ( $k+$ $1)$-slope theorem for the $k$-dimensional infinite group relaxation. eprint arXiv: 1109.4184 [math.OC], 2011.

4. Gérard Cornuéjols and Marco Molinaro. A 3-slope theorem for the infinite relaxation in the plane. Mathematical Programming, pages 1-23, 2012.

5. Santanu S. Dey and Jean-Philippe P. Richard. Facets of two-dimensional infinite group problems. Mathematics of Operations Research, 33(1):140-166, 2008.

6. Santanu S. Dey and Jean-Philippe P. Richard. Relations between facets of low- and high-dimensional group problems. Math. Program., 123(2):285-313, June 2010.

7. Santanu S. Dey, Jean-Philippe P. Richard, Yanjun Li, and Lisa A. Miller. On the extreme inequalities of infinite group problems. Math. Program., 121(1):145-170, June 2009.

8. Ralph E. Gomory. Some polyhedra related to combinatorial problems. Linear Algebra and its Applications, 2(4):451-558, 1969.

9. Ralph E. Gomory and Ellis L. Johnson. Some continuous functions related to corner polyhedra, I. Mathematical Programming, 3:23-85, 1972. 10.1007/BF01585008.

10. Ralph E. Gomory and Ellis L. Johnson. Some continuous functions related to corner polyhedra, II. Mathematical Programming, 3:359-389, 1972. 10.1007/BF01585008.

11. Ralph E. Gomory and Ellis L. Johnson. T-space and cutting planes. Mathematical Programming, 96:341-375, 2003. 10.1007/s10107-003-0389-3. 


\section{A Appendix}

\section{A.1 Equivariant perturbations}

In this section we outline the theory of equivariant perturbations for the infinite group problem, used first in 2 for the case $k=1$.

We consider a subgroup of the group $\operatorname{Aff}\left(\mathbb{R}^{k}\right)$ of invertible affine linear transformations of $\mathbb{R}^{k}$ as follows.

Definition A.1. For a point $\mathbf{r} \in \mathbb{R}^{k}$, define the reflection $\rho_{\mathbf{r}}: \mathbb{R}^{k} \rightarrow \mathbb{R}^{k}, \mathbf{x} \mapsto$ $\mathbf{r}-\mathbf{x}$. For a vector $t \in \mathbb{R}^{k}$, define the translation $\tau_{\mathbf{t}}: \mathbb{R}^{k} \rightarrow \mathbb{R}, \mathbf{x} \mapsto \mathbf{x}+\mathbf{t}$.

Given a set $R$ of points and a set $U$ of vectors, we will define the subgroup

$$
\Gamma=\left\langle\rho_{\mathbf{r}}, \tau_{\mathbf{t}} \mid \mathbf{r} \in R, \mathbf{t} \in U\right\rangle
$$

Let $\mathbf{r}, \mathbf{s}, \mathbf{w}, \mathbf{t} \in \mathbb{R}^{k}$. Each reflection is an involution: $\rho_{\mathbf{r}} \circ \rho_{\mathbf{r}}=i d$, two reflections give one translation: $\rho_{\mathbf{r}} \circ \rho_{\mathbf{s}}=\tau_{\mathbf{r}-\mathbf{s}}$. Thus, if we assign a character $\chi\left(\rho_{\mathbf{r}}\right)=-1$ to every reflection and $\chi\left(\tau_{\mathbf{t}}\right)=+1$ to every translation, then this extends to a group character of $\Gamma$, that is, a group homomorphism $\chi: \Gamma \rightarrow \mathbb{C}^{\times}$.

On the other hand, not all pairs of reflections need to be considered: $\rho_{\mathbf{s}} \circ \rho_{\mathbf{w}}=$ $\left(\rho_{\mathbf{s}} \circ \rho_{\mathbf{r}}\right) \circ\left(\rho_{\mathbf{r}} \circ \rho_{\mathbf{w}}\right)=\left(\rho_{\mathbf{r}} \circ \rho_{\mathbf{s}}\right)^{-1} \circ\left(\rho_{\mathbf{r}} \circ \rho_{\mathbf{w}}\right)$. Thus the subgroup $T=\operatorname{ker} \chi$ of translations in $\Gamma$ is generated as follows. Let $\mathbf{r}_{1} \in R$ be any of the reflection points; then

$$
T=\left\langle\tau_{\mathbf{r}-\mathbf{r}_{1}}, \tau_{\mathbf{t}} \mid \mathbf{r} \in R, \mathbf{t} \in U\right\rangle .
$$

It is normal in $\Gamma$, as it is stable by conjugation by any reflection: $\rho_{\mathbf{r}} \circ \tau_{\mathbf{t}} \circ \rho_{\mathbf{r}}^{-1}=$ $\tau_{-\mathbf{t}}$. If $\gamma \in \Gamma$ is not a translation, i.e., $\chi(\gamma)=-1$, then it is generated by an odd number of reflections, and thus can be written as $\gamma=\tau \rho_{\mathbf{r}_{1}}$ with $\tau \in T$. Thus $\Gamma / T=\left\langle\rho_{\mathbf{r}_{1}}\right\rangle$ is of order 2 . In short, we have the following lemma.

Lemma A.2. The group $\Gamma$ is the semidirect product $T \rtimes\left\langle\rho_{\mathbf{r}_{1}}\right\rangle$, where the (normal) subgroup of translations can be written as

$$
T=\left\{\tau_{\mathbf{t}} \mid \mathbf{t} \in \Lambda\right\}
$$

where $\Lambda$ is the additive subgroup

$$
\Lambda=\left\langle\mathbf{r}-\mathbf{r}_{1}, \mathbf{t} \mid \mathbf{r} \in R, \mathbf{t} \in U\right\rangle_{\mathbb{Z}} \subseteq \mathbb{R}^{k} .
$$

Definition A.3. A function $\psi: \mathbb{R}^{k} \rightarrow \mathbb{R}$ is called $\Gamma$-equivariant if it satisfies the equivariance formula

$$
\psi(\gamma(\mathbf{x}))=\chi(\gamma) \psi(\mathbf{x}) \quad \text { for } \mathbf{x} \in \mathbb{R} \text { and } \gamma \in \Gamma .
$$

We note that if $\Lambda$ is discrete, i.e., a lattice, then there is a way to construct continuous $\Gamma$-equivariant functions by defining them on a fundamental domain and extending them to all of $\mathbb{R}^{k}$ via the equivariance formula (2). The same is true for the case where $\Lambda$ is a mixed lattice, i.e., a direct sum of a lattice in a subspace and another subspace. We omit the details. 


\section{A.2 Polyhedral complexes $\mathcal{P}_{q}, \Delta \mathcal{P}_{q}$, and unimodularity}

We first comment that $\mathbf{f}$ must be a vertex of $\mathcal{P}_{q}$ of any minimal valid function. We omit the proof here as it is very similar to ([2], Lemma 2.1).

Lemma A.4. If $\pi$ is a minimal function, then $\mathbf{f} \in \frac{1}{q} \mathbb{Z}^{2}$.

Definition A.5. For $I, J, K \in \mathcal{P}_{q} \backslash\{\emptyset\}$, we say $(I, J, K)$ is a valid triple provided that the following occur:

i. $K \subseteq I \oplus J$,

ii. For all $\mathbf{u} \in I$ there exists $a \mathbf{v} \in J$ such that $\mathbf{u} \oplus \mathbf{v} \in K$,

iii. For all $\mathbf{v} \in J$ there exists $a \mathbf{u} \in I$ such that $\mathbf{u} \oplus \mathbf{v} \in K$,

Equivalently, a valid triple $(I, J, K)$ is characterized by the following property.

iv. Whenever $I^{\prime}, J^{\prime}, K^{\prime}$ are sets such that $I^{\prime} \subseteq I, J^{\prime} \subseteq J, K^{\prime} \subseteq K$ and $F(I, J, K)=F\left(I^{\prime}, J^{\prime}, K^{\prime}\right)$ we have that $I^{\prime}=I, J^{\prime}=J, K^{\prime}=K$.

The construction of $\mathcal{P}_{q}$ has convenient properties such as the following.

Lemma A.6. Let $I, J \in \mathcal{P}_{q}$. Then $I \oplus J$ and $I \ominus J$ are both unions of faces in $\mathcal{P}_{q}$.

Proof. By construction, for any face $K \in \mathcal{P}_{q}$, the set $\{\mathbf{x} \bmod \mathbf{1} \mid \mathbf{x} \in K\}$ is also a face in $\mathcal{P}_{q}$. Therefore we only need to show that the Minkowski sums $I+J$ and $I-J$ are unions of faces in $\mathcal{P}_{q}$. Let

$$
A=\left[\begin{array}{cccccc}
1 & -1 & 0 & 0 & 1 & -1 \\
0 & 0 & 1 & -1 & 1 & -1
\end{array}\right]^{T}
$$

Let $\mathbf{a}^{i}$ be the $i^{\text {th }}$ row vector of $A$. Then there exists vectors $\mathbf{b}^{1}, \mathbf{b}^{2}$ such that $I=$ $\left\{\mathbf{x} \mid A \mathbf{x} \leq \mathbf{b}^{1}\right\}, J=\left\{\mathbf{y} \mid A \mathbf{y} \leq \mathbf{b}^{2}\right\}$. Moreover, due to the total unimodularity of the matrix $A$, the right-hand side vectors $\mathbf{b}^{1}, \mathbf{b}^{2}$ can be chosen so that $\mathbf{b}^{1}, \mathbf{b}^{2}$ are tight, i.e.,

$$
\max _{\mathbf{x} \in I} \mathbf{a}^{i} \cdot \mathbf{x}=\mathbf{b}_{i}^{1}, \quad \max _{\mathbf{y} \in J} \mathbf{a}^{i} \cdot \mathbf{y}=\mathbf{b}_{i}^{2}
$$

and $\mathbf{b}^{1}, \mathbf{b}^{2} \in \frac{1}{q} \mathbb{Z}^{2}$.

We claim that $I+J=\left\{\mathbf{x} \mid A \mathbf{x} \leq \mathbf{b}^{1}+\mathbf{b}^{2}\right\}$. Clearly $I+J \subseteq\{\mathbf{x} \mid A \mathbf{x} \leq$ $\left.\mathbf{b}^{1}+\mathbf{b}^{2}\right\}$. We show the reverse direction. Let $K^{\prime}$ be a facet (edge) of $I+\bar{J}$. Then $K^{\prime}=I^{\prime}+J^{\prime}$, where $I^{\prime}$ is a face of $I$ and $J^{\prime}$ is a face of $J$. Without loss of generality, assume that $I^{\prime}$ is an edge; then $J^{\prime}$ is either a vertex or an edge. By well-known properties of Minkowski sums, the normal cone of $K^{\prime}$ is the intersection of the normal cones of $I^{\prime}$ in $I$ and $J^{\prime}$ in $J$. Thus $K^{\prime}$ has the same normal direction as the facet (edge) $I^{\prime}$. This proves that $I+J=\{\mathbf{x} \mid A \mathbf{x} \leq \mathbf{b}\}$ for some vector $\mathbf{b}$.

Let $\mathbf{x}^{*}, \mathbf{y}^{*}$ be maximizers in 3 Then $\mathbf{x}^{*}+\mathbf{y}^{*} \in I+J$. Then

$$
\mathbf{b}_{i}^{1}+\mathbf{b}_{i}^{2}=\mathbf{a}^{i} \cdot \mathbf{x}^{*}+\mathbf{a}^{i} \cdot \mathbf{y}^{*} \leq \max _{\mathbf{z} \in I+J} \mathbf{a}^{i} \cdot \mathbf{z} \leq \max _{\mathbf{x} \in I} \mathbf{a}^{i} \cdot \mathbf{x}+\max _{\mathbf{y} \in J} \mathbf{a}^{i} \cdot \mathbf{y}=\mathbf{b}_{i}^{1}+\mathbf{b}_{i}^{2} .
$$


Therefore, $\max _{\mathbf{z} \in I+J} \mathbf{a}^{i} \cdot \mathbf{z}=\mathbf{b}_{i}^{1}+\mathbf{b}_{i}^{2}$, which shows that every constraint $a_{i} \cdot \mathbf{z} \leq \mathbf{b}_{i}^{1}$ is met at equality, and therefore $I+J=\left\{\mathbf{x} \mid A \mathbf{x} \leq \mathbf{b}^{1}+\mathbf{b}^{2}\right\}$ and we conclude that $I+J$ must be a union of subsets in $\mathcal{P}_{q}$.

The case $I-J=\{\mathbf{z}-\mathbf{y} \mid \mathbf{z} \in K, \mathbf{y} \in J\}$ is shown similarly.

Lemma A.7. $E(\pi)=\bigcup\left\{F(I, J, K) \mid(I, J, K) \in E_{\max }\left(\pi, \mathcal{P}_{q}\right)\right\}$.

Proof. Clearly the right hand side is a subset of $E(\pi)$. We show $E(\pi)$ is a subset of the right hand side. Suppose $(\mathbf{x}, \mathbf{y}) \in E(\pi)$. Let $I, J, K \in \mathcal{P}_{q}$ be minimal faces by set inclusion containing $\mathbf{x}, \mathbf{y}$, and $\mathbf{x} \oplus \mathbf{y}$, respectively. We show that $(I, J, K)$ is a valid triple. By Lemma A.6. $I \oplus J$ is a union of faces in $\mathcal{P}_{q}$. Since $\mathbf{x} \oplus \mathbf{y} \in I \oplus J$ and $\mathbf{x} \oplus \mathbf{y} \in K$, we have that $K \cap(I+J) \neq \emptyset$, and in particular, is a union of faces of $\mathcal{P}_{q}$ containing $\mathbf{x}+\mathbf{y}$. Since $K$ was chosen to be a minimal such face in $\mathcal{P}_{q}$ containing $\mathbf{x} \oplus \mathbf{y}$, we have that $K \subseteq I \oplus J$.

Similarly, by Lemma A.6, $K \ominus J$ is also a union of sets in $\mathcal{P}_{q}$ containing $\mathbf{x}$. Since $I$ is a minimal set containing $\mathbf{x}$, it must be that $I \subseteq K \ominus J$. Therefore, for any $\mathbf{u} \in I$, there exists a $\mathbf{v} \in J$ such that $\mathbf{u} \oplus \mathbf{v} \in K$.

Similarly, we find that for any $\mathbf{v} \in J$, there exists a $\mathbf{u} \in I$ such that $\mathbf{u} \oplus \mathbf{v} \in K$.

Since $I, J, K$ were chosen to be minimal in $\mathcal{P}_{q}$, the triple satisfies criterion (iv) of being a valid triple. Hence, $(I, J, K)$ is a valid triple.

Next we argue that $(I, J, K) \in E\left(\pi, \mathcal{P}_{q}\right)$. This is because $\Delta \pi$ is affine in $F(I, J, K), \Delta \pi \geq 0,(\mathbf{x}, \mathbf{y}) \in \operatorname{rel} \operatorname{int}(F(I, J, K)), \Delta \pi(\mathbf{x}, \mathbf{y})=0$ and therefore $\left.\Delta \pi\right|_{F(I, J, K)}=0$, i.e., $(I, J, K) \in E\left(\pi, \mathcal{P}_{q}\right)$.

Lastly, if $(I, J, K)$ is not maximal in $E\left(\pi, \mathcal{P}_{q}\right)$, then there exists a maximal $\left(I^{\prime}, J^{\prime}, K^{\prime}\right)$ such that $F\left(I^{\prime}, J^{\prime}, K^{\prime}\right) \supset(I, J, K)$, namely, $(\mathbf{x}, \mathbf{y}) \in F\left(I^{\prime}, J^{\prime}, K^{\prime}\right)$.

Next we study the complex $\Delta \mathcal{P}_{q}$.

Proof (of Lemma 3.7). Since $F \in \Delta \mathcal{P}_{q}$, we can write $F$ using the system of inequalities $F=\left\{(\mathbf{x}, \mathbf{y}) \in \mathbb{R}^{4}: \hat{A}(\mathbf{x}, \mathbf{y}) \leq \mathbf{b}\right\}$ where $\mathbf{b} \in \frac{1}{q} \mathbb{Z}^{9}$, the matrix $A$ is given by

$$
A=\left[\begin{array}{lllllllll}
1 & 0 & 1 & 0 & 0 & 0 & 1 & 0 & 1 \\
0 & 1 & 1 & 0 & 0 & 0 & 0 & 1 & 1 \\
0 & 0 & 0 & 1 & 0 & 1 & 1 & 0 & 1 \\
0 & 0 & 0 & 0 & 1 & 1 & 0 & 1 & 1
\end{array}\right]^{T}
$$

and the matrix $\hat{A}$ differs from $A$ only by scaling each row individually by \pm 1 . (This inequality representation of $F$ will usually be redundant.) By checking every subdeterminant of the matrix $A$, it can be verified that $A$ is totally unimodular, and therefore $\hat{A}$ is also totally unimodular. Therefore, the polytope $q F=\left\{(\mathbf{x}, \mathbf{y}) \in \mathbb{R}^{4}: \hat{A}(\mathbf{x}, \mathbf{y}) \leq q \mathbf{b}\right\}$ has integral vertices in $\mathbb{Z}^{4}$.

It follows that $P$ has vertices in $\frac{1}{q} \mathbb{Z}^{4}$. Therefore, $\mathbf{x}, \mathbf{y} \in \frac{1}{q} \mathbb{Z}^{2}$ and therefore are vertices of $\mathcal{P}_{q}$. 


\section{A.3 Continuity results}

In this section we prove Theorem 2.1 on continuity. Although similar results appear in [10, we provide proofs of these facts to keep this paper more selfcontained. We first prove the following lemma.

Lemma A.8. If $\theta: \mathbb{R}^{k} \rightarrow \mathbb{R}$ is a subadditive function and $\limsup _{h \rightarrow 0} \frac{|\theta(\mathbf{h})|}{|\mathbf{h}|}=$ $L<\infty$, then $\theta$ is Lipschitz continuous with Lipschitz constant $L$.

Proof. Fix any $\delta>0$. Since $\limsup _{\mathbf{h} \rightarrow 0} \frac{|\theta(\mathbf{h})|}{|\mathbf{h}|}=L$, there exists $\epsilon>0$ such that for any $\mathbf{x}, \mathbf{y} \in \mathbb{R}^{k}$ satisfying $|\mathbf{x}-\mathbf{y}|<\epsilon, \frac{|\theta(\mathbf{x}-\mathbf{y})|}{|\mathbf{x}-\mathbf{y}|}<L+\delta$. By subadditivity, $|\theta(\mathbf{x}-\mathbf{y})| \geq|\theta(\mathbf{x})-\theta(\mathbf{y})|$ and so $\frac{|\theta(\mathbf{x})-\theta(\mathbf{y})|}{|\mathbf{x}-\mathbf{y}|}<L+\delta$ for all $\mathbf{x}, \mathbf{y} \in \mathbb{R}^{k}$ satisfying $|\mathbf{x}-\mathbf{y}|<\epsilon$. This immediately implies that for all $\mathbf{x}, \mathbf{y} \in \mathbb{R}, \frac{|\theta(\mathbf{x})-\theta(\mathbf{y})|}{|\mathbf{x}-\mathbf{y}|}<L+\delta$, by simply breaking the interval $[\mathbf{x}, \mathbf{y}]$ into equal subintervals of size at most $\epsilon$. Since the choice of $\delta$ was arbitrary, this shows that for every $\delta>0, \frac{|\theta(\mathbf{x})-\theta(\mathbf{y})|}{|\mathbf{x}-\mathbf{y}|}<L+\delta$ and therefore, $\frac{|\theta(\mathbf{x})-\theta(\mathbf{y})|}{|\mathbf{x}-\mathbf{y}|} \leq L$. Therefore, $\theta$ is Lipschitz continuous with Lipschitz constant $L$.

Proof (of Theorem 2.1). The minimality of $\pi^{1}, \pi^{2}$ is clear. Since we assume $\pi^{1}, \pi^{2} \geq 0, \pi=\frac{1}{2} \pi^{1}+\frac{1}{2} \pi^{2}$ implies that $\pi^{i} \leq 2 \pi$ for $i=1,2$. Therefore if $\limsup _{\mathbf{h} \rightarrow 0} \frac{|\pi(\mathbf{h})|}{|\mathbf{h}|}=L<\infty$, then $\limsup _{\mathbf{h} \rightarrow 0} \frac{\left|\pi^{i}(\mathbf{h})\right|}{|\mathbf{h}|} \leq 2 L<\infty$ for $i=1,2$. Applying Lemma A.8, we get Lipschitz continuity for all three functions.

The following is a slight generalization of the Interval Lemma that appears in [1. The proof is a minor modification of the original proof.

\section{A.4 Finite test for minimality of piecewise linear functions}

In this subsection, we show that there is an easy test to see if a continuous piecewise linear function over $\mathcal{P}_{q}$ is minimal.

Lemma A.9. Suppose that $\pi$ is a continuous piecewise linear function over $\mathcal{P}_{q}$ and $\pi(\mathbf{0})=0$.

1. $\pi$ is subadditive if and only if $\pi(\mathbf{x})+\pi(\mathbf{y}) \geq \pi(\mathbf{x} \oplus \mathbf{y})$ for all $\mathbf{x}, \mathbf{y} \in \frac{1}{q} \mathbb{Z}^{2}$,

2. $\pi$ is symmetric if and only if $\pi(\mathbf{x})+\pi(\mathbf{f} \ominus \mathbf{x})=1$ for all $\mathbf{x} \in \frac{1}{q} \mathbb{Z}^{2}$.

Proof. Clearly the forward direction of both statements is true. We will show the reverse of each. For subadditivity, we need to show that $\Delta \pi \geq 0$. Since $\Delta \pi$ is piecewise linear over $\Delta \mathcal{P}_{q}$, we just need to show that $\Delta \pi(\mathbf{x}, \mathbf{y}) \geq 0$ for any $(\mathbf{x}, \mathbf{y}) \in \operatorname{vert}\left(\Delta \mathcal{P}_{q}\right)$. By Lemma 3.7, $\operatorname{vert}\left(\Delta \mathcal{P}_{q}\right) \subseteq \frac{1}{q} \mathbb{Z}^{4}$, and the result follows.

Next, we show symmetry. Since $\mathbf{0}, \mathbf{f} \in \frac{1}{q} \mathbb{Z}^{2}$ and $\pi(\mathbf{0})=0$, we have that $\pi(\mathbf{f})=1$. Let $\mathbf{x} \in[0,1]^{2}$ and let $F \in \Delta \mathcal{P}_{q}$ such that $(\mathbf{x}, \mathbf{f} \ominus \mathbf{x}) \in F$.

Similarly, to show symmetry, we need to show that $\Delta \pi(\mathbf{x}, \mathbf{y})=0$ for all $\mathbf{x}, \mathbf{y} \in[0,1]^{2}$ such that $\mathbf{x} \oplus \mathbf{y}=\mathbf{f}$. Let $\mathbf{x}, \mathbf{y} \in[0,1]^{2}$ such that $\mathbf{x} \oplus \mathbf{y}=\mathbf{f}$. Since 
$\mathbf{f} \in \frac{1}{q} \mathbb{Z}^{2}$ by Lemma A.4. $(\mathbf{x}, \mathbf{y}) \in \operatorname{rel} \operatorname{int}(\hat{F})$ for some face $\hat{F}$ of some $F \in \Delta \mathcal{P}_{q}$ and $\hat{F} \subseteq\{(\mathbf{x}, \mathbf{y}) \mid \mathbf{x} \oplus \mathbf{y}=f\}$. Since $\Delta \pi_{F}(\mathbf{u}, \mathbf{v})=0$ for all $(\mathbf{u}, \mathbf{v}) \in \operatorname{vert}(F) \subset \frac{1}{q} \mathbb{Z}^{2}$ when $\mathbf{u} \oplus \mathbf{v}=\mathbf{f}$, and $\Delta \pi_{F}$ is affine, it follows that $\Delta \pi(\mathbf{x}, \mathbf{y})=\Delta \pi_{F}(\mathbf{x}, \mathbf{y})=0$.

The following theorem is a direct corollary of Lemma A.9 and Theorem 1.1 .

Theorem A.10 (Minimality test). A function $\pi: \mathbb{R}^{2} \rightarrow \mathbb{R}$ that is continuous piecewise linear over $\mathcal{P}_{q}$ is minimal if and only if

1. $\pi(\mathbf{0})=0$,

2. $\pi(\mathbf{x})+\pi(\mathbf{y}) \geq \pi(\mathbf{x} \oplus \mathbf{y})$ for all $\mathbf{x}, \mathbf{y} \in \frac{1}{q} \mathbb{Z}^{2}$,

3. $\pi(\mathbf{x})+\pi(\mathbf{f} \ominus \mathbf{x})=1$ for all $\mathbf{x} \in \frac{1}{q} \mathbb{Z}^{2}$.

\section{A.5 Properties of valid triples}

Lemma A.11. Suppose $\pi$ is continuous piecewise linear over $\mathcal{P}_{q}$ and is diagonally constrained. Suppose that $(I, J, K) \in E\left(\pi, \mathcal{P}_{q}\right)$. Then one of the following is true.

1. $I, J, K \in \mathcal{P}_{q, 0} \cup \mathcal{P}_{q, \backslash}$,

2. $I, J, K \in \mathcal{P}_{q, 2}$,

3. One of $I, J, K$ is in $\mathcal{P}_{q, 0}$, while the other two are in $\mathcal{P}_{q, 2}$,

4. One of $I, J, K$ is in $\mathcal{P}_{q, \backslash}$, while the other two are in $\mathcal{P}_{q, 2}$

Proof. By definition of diagonally constrained, $I, J, K \in \mathcal{P}_{q, 0} \cup \mathcal{P}_{q, \backslash} \cup \mathcal{P}_{q, 2}$. There are 27 possible ways to put $I, J, K$ into those three sets. Above, 15 possibilities are described. We will show that the 12 remaining cases not list above are not possible because $(I, J, K)$ is assumed to be a valid triple.

1. Suppose $I, J \in \mathcal{P}_{q, 0} \cup \mathcal{P}_{q, \backslash}, K \in I_{q, 2}$. Then $K^{\prime}=I \oplus J \subsetneq K$, and therefore $F(I, J, K)=F\left(I, J, K^{\prime}\right)$, and therefore $(I, J, K)$ is not a valid triple.

2. Suppose $I, K \in \mathcal{P}_{q, 0} \cup \mathcal{P}_{q, \backslash}, J \in I_{q, 2}$. Then $K \ominus I \subsetneq J$, and therefore, there exists a $J^{\prime} \subsetneq J$ such that $F(I, J, K)=F\left(I, J^{\prime}, K\right)$, and therefore $(I, J, K)$ is not a valid triple.

3. Suppose $J, K \in \mathcal{P}_{q, 0} \cup \mathcal{P}_{q, \backslash}, I \in I_{q, 2}$. This is similar to the last case.

Lemma A.12. Suppose $(I, J, K)$ is a valid triple. The following are true.

i. Suppose $I, J \in \mathcal{P}_{q, 2}$. Then for every point $\mathbf{u} \in \operatorname{int}(I)$ there exists a point $\mathbf{v} \in \operatorname{int}(J)$ such that $\mathbf{u} \oplus \mathbf{v} \in \operatorname{rel} \operatorname{int}(K)$.

ii. Suppose $I, K \in \mathcal{P}_{q, 2}$. Then for every point $\mathbf{w} \in \operatorname{int}(K)$ there exists a point $\mathbf{u} \in \operatorname{int}(I)$ such that $\mathbf{w} \ominus \mathbf{u} \in \operatorname{rel} \operatorname{int}(J)$.

Proof. Part (i). Let $\mathbf{u} \in \operatorname{int}(I)$ and so $(1,0)^{T} \mathbf{u},(0,1)^{T} \mathbf{u}$ and $(1,1)^{T} \mathbf{u}$ are all nonzero modulo $\frac{1}{q}$. Since $(I, J, K)$ is a valid triple, there exist $\mathbf{v} \in J$ and $\mathbf{w} \in K$ such that $\mathbf{u} \oplus \mathbf{v}=\mathbf{w}$. Thus, $(1,0)^{T} \mathbf{v}$ and $(1,0)^{T} \mathbf{w}$ are different modulo $\frac{1}{q}$ (resp. for $(0,1)^{T} \mathbf{v},(0,1)^{T} \mathbf{w}$ and $\left.(1,1)^{T} \mathbf{v},(1,1)^{T} \mathbf{w}\right)$. Note that for any point $\mathbf{x} \in \mathbb{R}^{2}$, 
either $(1,0)^{T} \mathbf{x},(0,1)^{T} \mathbf{x}$ and $(1,1)^{T} \mathbf{x}$ are all 0 modulo $\frac{1}{q}$, or exactly one of these numbers is 0 modulo $\frac{1}{q}$, or none of them are 0 . Thus, we consider these cases :

Case 1: $(1,0)^{T} \mathbf{w},(0,1)^{T} \mathbf{w}$ and $(1,1)^{T} \mathbf{w}$ are all 0 modulo $\frac{1}{q}$. Then $\mathbf{v} \in \operatorname{int}(J)$ since $J \in \mathcal{P}_{q, 2}$. Then one can choose a vector $\mathbf{d}$ such that $\mathbf{w}^{\prime}=\mathbf{w}+\mathbf{d} \in \operatorname{rel} \operatorname{int}(K)$ and $\mathbf{v}^{\prime}=\mathbf{v}+\mathbf{d} \in \operatorname{int}(J)$. Then $\mathbf{u} \oplus \mathbf{v}^{\prime}=\mathbf{w}^{\prime}$ and we are done.

Case 2: $(1,0)^{T} \mathbf{v},(0,1)^{T} \mathbf{v}$ and $(1,1)^{T} \mathbf{v}$ are all 0 modulo $\frac{1}{q}$. Then $\mathbf{w} \in \operatorname{int}(K)$ and one can choose again a vector $\mathbf{d}$ such that $\mathbf{w}^{\prime}=\mathbf{w}+\mathbf{d} \in \operatorname{int}(K)$ and $\mathbf{v}^{\prime}=\mathbf{v}+\mathbf{d} \in \operatorname{int}(J)$. Then $\mathbf{u} \oplus \mathbf{v}^{\prime}=\mathbf{w}^{\prime}$ and we are done.

Case 3: Exactly one of $(1,0)^{T} \mathbf{w},(0,1)^{T} \mathbf{w}$ and $(1,1)^{T} \mathbf{w}$ is 0 modulo $\frac{1}{q}$ and the same holds for $\mathbf{v}$. This means $\mathbf{w}$ and $\mathbf{v}$ lie on different hyperplanes in the arrangement $\mathcal{H}_{q}$. But then one can again choose a vector $\mathbf{d}$ such that $\mathbf{w}^{\prime}=$ $\mathbf{w}+\mathbf{d} \in \operatorname{rel} \operatorname{int}(K)$ and $\mathbf{v}^{\prime}=\mathbf{v}+\mathbf{d} \in \operatorname{int}(J)$. Then $\mathbf{u} \oplus \mathbf{v}^{\prime}=\mathbf{w}^{\prime}$ and we are done.

Case 4: None of $(1,0)^{T} \mathbf{w},(0,1)^{T} \mathbf{w}$ and $(1,1)^{T} \mathbf{w}$ is 0 modulo $\frac{1}{q}$ and the same holds for $\mathbf{v}$. This means $\mathbf{v} \in \operatorname{int}(J)$ and $\mathbf{w} \in \operatorname{int}(K)$ already and we are done.

Part (ii) can be proved in a similar way.

\section{A.6 Interval lemma}

The so-called Interval Lemma was introduced by Gomory and Johnson in [11]. We prove this in a more general setting with three functions by a modifying a proof from [1].

Lemma A.13 (Interval Lemma). Given real numbers $u_{1}<u_{2}$ and $v_{1}<v_{2}$, let $U=\left[u_{1}, u_{2}\right], V=\left[v_{1}, v_{2}\right]$, and $U+V=\left[u_{1}+v_{1}, u_{2}+v_{2}\right]$. Let $f: U \rightarrow \mathbb{R}$, $g: V \rightarrow \mathbb{R}, h: U+V \rightarrow \mathbb{R}$ be bounded functions.

If $f(u)+g(v)=h(u+v)$ for every $u \in U$ and $v \in V$, then there exists $c \in \mathbb{R}$ such that $f(u)=f\left(u_{1}\right)+c\left(u-u_{1}\right)$ for every $u \in U, g(v)=g\left(v_{1}\right)+c\left(v-v_{1}\right)$ for every $v \in V, h(w)=h\left(u_{1}+v_{1}\right)+c\left(w-u_{1}-v_{1}\right)$ for every $w \in U+V$.

Proof. We first show the following.

Claim 1. Let $u \in U$, and let $\varepsilon>0$ such that $v_{1}+\varepsilon \in V$. For every nonnegative integer $p$ such that $u+p \varepsilon \in U$, we have $f(u+p \varepsilon)-f(u)=p\left(g\left(v_{1}+\varepsilon\right)-g\left(v_{1}\right)\right)$.

For $h=1, \ldots, p$, by hypothesis $f(u+h \varepsilon)+g\left(v_{1}\right)=h\left(u+h \varepsilon+v_{1}\right)=f(u+$ $(h-1) \varepsilon)+g\left(v_{1}+\varepsilon\right)$. Thus $f(u+h \varepsilon)-f(u+(h-1) \varepsilon)=g\left(v_{1}+\varepsilon\right)-g\left(v_{1}\right)$, for $h=1, \ldots, p$. By summing the above $p$ equations, we obtain $f(u+p \varepsilon)-f(u)=$ $p\left(g\left(v_{1}+\varepsilon\right)-g\left(v_{1}\right)\right)$. This concludes the proof of Claim 1 .

Let $\bar{u}, \bar{u}^{\prime} \in U$ such that $\bar{u}-\bar{u}^{\prime} \in \mathbb{Q}$ and $\bar{u}>\bar{u}^{\prime}$. Define $c:=\frac{f(\bar{u})-f\left(\bar{u}^{\prime}\right)}{\bar{u}-\bar{u}^{\prime}}$.

Claim 2. For every $u, u^{\prime} \in U$ such that $u-u^{\prime} \in \mathbb{Q}$, we have $f(u)-f\left(u^{\prime}\right)=$ $c\left(u-u^{\prime}\right)$.

We only need to show that, given $u, u^{\prime} \in U$ such that $u-u^{\prime} \in \mathbb{Q}$, we have $f(u)-f\left(u^{\prime}\right)=c\left(u-u^{\prime}\right)$. We may assume $u>u^{\prime}$. Choose a positive rational $\varepsilon$ 
such that $\bar{u}-\bar{u}^{\prime}=\bar{p} \varepsilon$ for some integer $\bar{p}, u-u^{\prime}=p \varepsilon$ for some integer $p$, and $v_{1}+\varepsilon \in V$. By Claim 1,

$$
f(\bar{u})-f\left(\bar{u}^{\prime}\right)=\bar{p}\left(g\left(v_{1}+\varepsilon\right)-g\left(v_{1}\right)\right) \quad \text { and } \quad f(u)-f\left(u^{\prime}\right)=p\left(g\left(v_{1}+\varepsilon\right)-g\left(v_{1}\right)\right) .
$$

Dividing the last equality by $u-u^{\prime}$ and the second to last by $\bar{u}-\bar{u}^{\prime}$, we get

$$
\frac{g\left(v_{1}+\varepsilon\right)-g\left(v_{1}\right)}{\varepsilon}=\frac{f(\bar{u})-f\left(\bar{u}^{\prime}\right)}{\bar{u}-\bar{u}^{\prime}}=\frac{f(u)-f\left(u^{\prime}\right)}{u-u^{\prime}}=c .
$$

Thus $f(u)-f\left(u^{\prime}\right)=c\left(u-u^{\prime}\right)$. This concludes the proof of Claim 2 .

Claim 3. For every $u \in U, f(u)=f\left(u_{1}\right)+c\left(u-u_{1}\right)$.

Let $\delta(x)=f(x)-c x$ for all $x \in U$. We show that $\delta(u)=\delta\left(u_{1}\right)$ for all $u \in U$ and this proves the claim. Since $f$ is bounded on $U, \delta$ is bounded over $U$. Let $M$ be a number such that $|\delta(x)| \leq M$ for all $x \in U$.

Suppose by contradiction that, for some $u^{*} \in U, \delta\left(u^{*}\right) \neq \delta\left(u_{1}\right)$. Let $N$ be a positive integer such that $\left|N\left(\delta\left(u^{*}\right)-\delta\left(u_{1}\right)\right)\right|>2 M$.

By Claim 2, $\delta\left(u^{*}\right)=\delta(u)$ for every $u \in U$ such that $u^{*}-u$ is rational. Thus there exists $\bar{u}$ such that $\delta(\bar{u})=\delta\left(u^{*}\right), u_{1}+N\left(\bar{u}-u_{1}\right) \in U$ and $v_{1}+\bar{u}-u_{1} \in V$. Let $\bar{u}-u_{1}=\varepsilon$. By Claim 1 ,

$$
\begin{aligned}
\delta\left(u_{1}+N \varepsilon\right)-\delta\left(u_{1}\right) & =\left(f\left(u_{1}+N \varepsilon\right)-c\left(u_{1}+N \varepsilon\right)\right)-\left(f\left(u_{1}\right)-c u_{1}\right) \\
& =N\left(g\left(v_{1}+\varepsilon\right)-g\left(v_{1}\right)\right)-c(N \varepsilon) \\
& =N\left(f\left(u_{1}+\varepsilon\right)-f\left(u_{1}\right)\right)-c(N \varepsilon) \\
& =N\left(f\left(u_{1}+\varepsilon\right)-f\left(u_{1}\right)-c \varepsilon\right) \\
& =N\left(\delta\left(u_{1}+\varepsilon\right)-\delta\left(u_{1}\right)\right) \\
& =N\left(\delta(\bar{u})-\delta\left(u_{1}\right)\right)
\end{aligned}
$$

Thus $\left|\delta\left(u_{1}+N \varepsilon\right)-\delta\left(u_{1}\right)\right|=\left|N\left(\delta(\bar{u})-\delta\left(u_{1}\right)\right)\right|=\left|N\left(\delta\left(u^{*}\right)-\delta\left(u_{1}\right)\right)\right|>2 M$, which implies $\left|\delta\left(u_{1}+N \varepsilon\right)\right|+\left|\delta\left(u_{1}\right)\right|>2 M$, a contradiction. This concludes the proof of Claim 3 .

By symmetry between $U$ and $V$, Claim 3 implies that there exists some constant $c^{\prime}$ such that, for every $v \in V, g(v)=g\left(v_{1}\right)+c^{\prime}\left(v-v_{1}\right)$. We show $c^{\prime}=c$. Indeed, given $\varepsilon>0$ such that $u_{1}+\varepsilon \in U$ and $v_{1}+\varepsilon \in V, c \varepsilon=f\left(u_{1}+\varepsilon\right)-f\left(u_{1}\right)=$ $g\left(v_{1}+\varepsilon\right)-g\left(v_{1}\right)=c^{\prime} \varepsilon$, where the second equality follows from Claim 1 .

Therefore, for every $v \in V, g(v)=g\left(v_{1}\right)+c g\left(v-v_{1}\right)$. Finally, since $f(u)+g(v)=$ $h(u+v)$ for every $u \in U$ and $v \in V$, we have that for every $w \in U+V$, $h(w)=h\left(u_{1}+v_{1}\right)+c\left(w-u_{1}-v_{1}\right)$.

\section{A.7 Generalized interval lemma and corollaries}

The following lemma is a generalization to higher dimensions of the interval lemma that appears in the literature for the infinite group problem. 
Lemma A.14 (Higher Dimensional Interval Lemma). Let $\pi: \mathbb{R}^{k} \rightarrow \mathbb{R}$ be a bounded function. Let $U$ and $V$ be compact convex subsets of $\mathbb{R}^{k}$ such that $\pi(\mathbf{u})+\pi(\mathbf{v})=\pi(\mathbf{u}+\mathbf{v})$ for all $\mathbf{u} \in U$ and $\mathbf{v} \in V$. Corresponding to every linear subspace $L$ of $\mathbb{R}^{k}$, there exists a vector $\mathbf{g}$ in the dual space $L^{\prime}$ of $L$ with the following property. For any $\mathbf{u}^{0} \in U$ and $\mathbf{v}^{0} \in V$ such that $\mathbf{u}^{0}$ (resp. $\mathbf{v}^{0}$ ) is in the interior of $\left(\mathbf{u}^{0}+L\right) \cap U$ (resp. $\left(\mathbf{v}^{0}+L\right) \cap V$ ) in the relative topology of L, the following conditions hold:

(i) $\pi\left(\mathbf{u}^{0}+\mathbf{p}\right)=\pi\left(\mathbf{u}^{0}\right)+\langle\mathbf{g}, \mathbf{p}\rangle$ for all $\mathbf{p} \in L$ such that $\mathbf{u}^{0}+\mathbf{p} \in U$.

(ii) $\pi\left(\mathbf{v}^{0}+\mathbf{p}\right)=\pi\left(\mathbf{v}^{0}\right)+\langle\mathbf{g}, \mathbf{p}\rangle$ for all $\mathbf{p} \in L$ such that $\mathbf{v}^{0}+\mathbf{p} \in V$.

(iii) $\pi\left(\mathbf{u}^{0}+\mathbf{v}^{0}+\mathbf{p}\right)=\pi\left(\mathbf{u}^{0}+\mathbf{v}^{0}\right)+\langle\mathbf{g}, \mathbf{p}\rangle$ for all $\mathbf{p} \in L$ such that $\mathbf{v}^{0}+\mathbf{p} \in U+V$.

Proof. We fix an arbitrary linear subspace $L$ and exhibit a vector $\mathbf{g} \in L^{\prime}$ with the stated property. Let $\mathbf{p}^{1}, \ldots, \mathbf{p}^{m}$ be a basis for $L$ (we obviously have $m \leq k$ ). Now consider any $\mathbf{u}^{0} \in U$ and $\mathbf{v}^{0} \in V$ such that $\mathbf{u}^{0}$ (resp. $\mathbf{v}^{0}$ ) is in the interior of $\left(\mathbf{u}^{0}+L\right) \cap U$ (resp. $\left.\left(\mathbf{v}^{0}+L\right) \cap V\right)$ in the relative topology of $L$. Let $u_{1}^{i}<u_{2}^{i} \in \mathbb{R}$, $i=1, \ldots, m$ be such that the intersection of the line $\mathbf{u}^{0}+\lambda \mathbf{p}^{i}$ with $U$ is given by $\left\{u^{0}+\lambda p: u_{1}^{i} \leq \lambda \leq u_{2}^{i}\right\}$ (these numbers exist since $U$ is assumed to be compact and convex), similarly, $v_{1}^{i}<v_{2}^{i} \in \mathbb{R}$ are defined with respect to $V, \mathbf{v}^{0}$ and $\mathbf{p}^{i}$.

Let $f^{i}:\left[u_{1}^{i}, u_{2}^{i}\right] \rightarrow \mathbb{R}$ be defined by $f^{i}(\lambda)=\pi\left(\mathbf{u}^{0}+\lambda \mathbf{p}^{i}\right), g^{i}:\left[v_{1}^{i}, v_{2}^{i}\right] \rightarrow \mathbb{R}$ be defined by $g^{i}(\lambda)=\pi\left(\mathbf{v}^{0}+\lambda \mathbf{p}^{i}\right)$ and $h^{i}:\left[u_{1}^{i}+v_{1}^{i}, u_{2}^{i}+v_{2}^{i}\right] \rightarrow \mathbb{R}$ be defined by $h^{i}(\lambda)=\pi\left(\mathbf{u}^{0}+\mathbf{v}^{0}+\lambda \mathbf{p}^{i}\right)$. Applying Lemma A.13 there exists a constant $c_{i} \in \mathbb{R}$ such that

$$
\begin{aligned}
& \pi\left(\mathbf{u}^{0}+\lambda \mathbf{p}^{i}\right)=\pi\left(\mathbf{u}^{0}\right)+c_{i} \cdot \lambda \text { for all } \lambda \in\left[u_{1}^{i}, u_{2}^{i}\right] \\
& \pi\left(\mathbf{v}^{0}+\lambda \mathbf{p}^{i}\right)=\pi\left(\mathbf{u}^{0}\right)+c_{i} \cdot \lambda \text { for all } \lambda \in\left[v_{1}^{i}, v_{2}^{i}\right] \text { and } \\
& \pi\left(\mathbf{u}^{0}+\mathbf{v}^{0}+\lambda \mathbf{p}^{i}\right)=\pi\left(\mathbf{u}^{0}+\mathbf{v}^{0}\right)+c_{i} \cdot \lambda \text { for all } \lambda \in\left[u_{1}^{i}+v_{1}^{i}, u_{2}^{i}+v_{2}^{i}\right] .
\end{aligned}
$$

Notice that this argument could be made with $\mathbf{u}^{0}$ and any other $\mathbf{v} \in V$ with the property that $\mathbf{v}$ is in the interior of $(\mathbf{v}+L) \cap V$. Thus, $c_{i}$ is independent of $\mathbf{v}^{0}$. Applying a symmetric argument by fixing $\mathbf{v}^{0}$ and considering different $\mathbf{u} \in U$, we see that $c_{i}$ is also independent of $\mathbf{u}^{0}$. In other words, $c_{i}, i=1, \ldots, m$ only depend on $\pi, L$ and the two sets $U$ and $V$, and (4) holds for any $\mathbf{u} \in U$ and $\mathbf{v} \in V$ with the property that $\mathbf{u}$ (resp. $\mathbf{v}$ ) is in the interior of $(\mathbf{u}+L) \cap V$ (resp. $(\mathbf{u}+L) \cap V)$ in the relative topology of $L$.

We choose $\mathbf{g} \in L^{\prime}$ as the unique dual vector satisfying $\left\langle\mathbf{g}, \mathbf{p}^{i}\right\rangle=c_{i}$. Now for any $\mathbf{p} \in L$ such that $\mathbf{u}^{0}+\mathbf{p} \in U$. We can then represent $\mathbf{p}=\sum_{i=1}^{m} \lambda_{i} \mathbf{p}^{i}$ such that $u_{1}^{i} \leq \lambda_{i} \leq u_{2}^{i}$. Thus, $\pi\left(\mathbf{u}^{0}+\mathbf{p}\right)=\pi\left(\mathbf{u}^{0}+\sum_{i=1}^{m} \lambda_{i} \mathbf{p}^{i}\right)$. Now using (4) with $i=m$ we have

$$
\begin{aligned}
\pi\left(\mathbf{u}^{0}+\sum_{i=1}^{m} \lambda_{i} \mathbf{p}^{i}\right) & =\pi\left(\mathbf{u}^{0}+\sum_{i=1}^{m-1} \lambda_{i} \mathbf{p}^{i}+\lambda_{m} \mathbf{p}^{m}\right) \\
& =\pi\left(\mathbf{u}^{0}+\sum_{i=1}^{m-1} \lambda_{i} \mathbf{p}^{i}\right)+c_{m} \cdot \lambda_{m}
\end{aligned}
$$

which follows because the $c_{i}$ 's do not depend on the particular point $\mathbf{u}^{0}$ and in the case above we apply it on the point $\mathbf{u}^{0}+\sum_{i=1}^{m-1} \lambda_{i} \mathbf{p}^{i}$. By applying this argument iteratively, we find that 


$$
\begin{aligned}
\pi\left(\mathbf{u}^{0}+\sum_{i=1}^{m} \lambda_{i} \mathbf{p}^{i}\right) & =\pi\left(\mathbf{u}^{0}\right)+\sum_{i=1}^{m} c_{i} \cdot \lambda_{i} \\
& =\pi\left(\mathbf{u}^{0}\right)+\sum_{i=1}^{m}\left\langle\mathbf{g}, \mathbf{p}^{i}\right\rangle \cdot \lambda_{i} \\
& =\pi\left(\mathbf{u}^{0}\right)+\left\langle\mathbf{g}, \sum_{i=1}^{m} \lambda_{i} \mathbf{p}^{i}\right\rangle \\
& =\pi\left(\mathbf{u}^{0}\right)+\langle\mathbf{g}, \mathbf{p}\rangle
\end{aligned}
$$

This proves condition $(i)$ in the statement of the lemma. The same argument applies for proving conditions (ii) and (iii).

Now the lemmas stated in section 2 follow as corollaries.

Proof (of Lemma 2.2). Let $U(\mathbf{x}, r) \subseteq \mathbb{R}^{2}$ denote the $\ell_{\infty}$ ball of radius $r$ around $\mathbf{x} \in \mathbb{R}^{2}$. Define

$$
r(\mathbf{u})=\sup \{r \in \mathbb{R}: \exists \mathbf{v}, \mathbf{w} \text { such that } U(\mathbf{u}, r) \subseteq I, U(\mathbf{v}, r) \subseteq J, U(\mathbf{w}, 2 r) \subseteq K\} .
$$

Since $(I, J, K)$ is a valid triple, by Lemma A.12 (i), for any $\mathbf{u} \in \operatorname{int}(I)$, there exist $\mathbf{v} \in \operatorname{int}(J)$ and $\mathbf{w} \in \operatorname{int}(K)$ such that $\mathbf{u} \oplus \mathbf{v}=\mathbf{w}$. Thus, $r(\mathbf{u})>0$ for every $\mathbf{u} \in I$.

Claim. $r(\mathbf{u})$ is a continuous function of $\mathbf{u}$.

Proof. $r(\mathbf{u})$ is the optimal value of the linear program with variables $r, \mathbf{v}, \mathbf{w}$ given by

$\max r$ subject to $\mathbf{u} \oplus \mathbf{v}=\mathbf{w}, U(\mathbf{u}, r) \subseteq I, U(\mathbf{v}, r) \subseteq J, U(\mathbf{w}, 2 r) \subseteq K$.

All the constraints can be written as linear constraints. Since the value of a parametric linear program is continuous in the parameter (in this case the parameter is $\mathbf{u}$ ) we are done.

We will now show that for any two points $\mathbf{x}_{1}, \mathbf{x}_{2} \in \operatorname{int}(I)$, there exist finitely many full-dimensional parallelotopes $U_{1}, \ldots, U_{k}$ in $\mathbb{R}^{2}$ such that $\mathbf{x}_{1} \in U_{1}, \mathbf{x}_{2} \in$ $U_{k}$ and $\operatorname{int}\left(U_{i}\right) \cap \operatorname{int}\left(U_{i+1}\right) \neq \emptyset$ for all $i=1, \ldots, k-1$. Moreover, we will show that $\pi$ is affine over each $U_{i}, i=1, \ldots, k$. This will imply that in fact $\pi$ is affine over $\operatorname{int}(I)$ and therefore, by continuity, over $I$. By a symmetric argument, one can show that $\pi$ is affine over $J$. This will then show that $\pi$ is affine over $K$.

Given $\mathbf{x}_{1}, \mathbf{x}_{2} \in \operatorname{int}(I)$, consider the minimum value $\epsilon$ of $r(\mathbf{u})$ as $\mathbf{u}$ varies over the line segment $\left[\mathbf{x}_{1}, \mathbf{x}_{2}\right]$. Note that $\epsilon$ is strictly greater than 0 as it is the minimum of a strictly positive function over a compact set. This implies that we can find a set of points $\mathbf{u}_{1}=\mathbf{x}_{1}, \ldots, \mathbf{u}_{k}=\mathbf{x}_{2}$ on the line segment $\left[\mathbf{x}_{1}, \mathbf{x}_{2}\right]$ such that if we let $U_{i}=U\left(\mathbf{u}_{i}, \epsilon\right)$ we have the property that $\mathbf{x}_{1} \in U_{1}, \mathbf{x}_{2} \in U_{k}$ and $\operatorname{int}\left(U_{i}\right) \cap \operatorname{int}\left(U_{i+1}\right) \neq \emptyset$ for all $i=1, \ldots, k-1$. Now, by the definition of $r\left(\mathbf{u}_{i}\right)$ which is greater than or equal to $\epsilon$, there exist $\mathbf{v}_{i}$ and $\mathbf{w}_{i}, i=1, \ldots, k$ such that $U\left(\mathbf{u}_{i}, r\left(u_{i}\right)\right) \in I, U\left(\mathbf{v}_{i}, r\left(\mathbf{u}_{i}\right)\right) \in J, U\left(\mathbf{w}_{i}, 2 r\left(\mathbf{u}_{i}\right)\right) \in K$. Applying Lemma A.14. with $L=\mathbb{R}^{2}, U=U\left(\mathbf{u}_{i}, r\left(\mathbf{u}_{i}\right)\right), V=U\left(\mathbf{v}_{i}, r\left(\mathbf{u}_{i}\right)\right)$ and $\mathbf{u}_{0}=\mathbf{u}_{i}$ and $\mathbf{v}_{0} \in v_{i}$, we obtain that $\pi$ is affine over $U\left(\mathbf{u}_{i}, r\left(\mathbf{u}_{i}\right)\right)$ and hence over $U_{i} \subseteq U\left(\mathbf{u}_{i}, r\left(\mathbf{u}_{i}\right)\right)$.

The fact that the gradient over $I$ and $J$ (and hence over $K$ ) are the same follows from the observation that Lemma A.14 gives the same gradient over the parallelotopes $U=U\left(\mathbf{u}_{i}, r\left(\mathbf{u}_{i}\right)\right)$ and $V=\overline{U\left(\mathbf{v}_{i}, r\left(\mathbf{u}_{i}\right)\right)}$ in the above argument.

Similar arguments can be used to show Lemma 2.3. We omit the proof. 


\section{A.8 Transferring affine linearity}

Proof (of Lemma 2.4). Let $e \in \mathcal{P}_{q, 1}$ be the common edge for $I$ and $J$. We assume that $e$ is horizontal (the argument for vertical edges is exactly the same) and let $\mathbf{v}^{0} \in \mathbb{R}^{2}$ be the vertex of $e$ such that the other vertex is $\mathbf{v}^{0}+(0,1)^{T}$. Since $\pi$ is affine on $I$, there exists $c^{\prime} \in \mathbb{R}$ such that $\pi\left(\mathbf{v}^{0}+\lambda(0,1)^{T}\right)=\pi\left(\mathbf{v}^{0}\right)+c^{\prime} \cdot \lambda$ for all $0 \leq \lambda \leq 1$. Now observe that any point in $J$ can be written as $\mathbf{v}^{0}+\mu_{1}(0,1)^{T}+$ $\mu_{2}(-1,1)^{T}$ with $0 \leq \mu_{1}, \mu_{2} \leq 1$ and therefore, $\pi\left(\mathbf{v}^{0}+\mu_{1}(0,1)^{T}+\mu_{2}(-1,1)^{T}\right)=$ $\pi\left(\mathbf{v}^{0}+\mu_{1}(0,1)^{T}\right)+c \cdot \mu_{2}$ (using (ii) in the hypothesis) and $\pi\left(\mathbf{v}^{0}+\mu_{1}(0,1)^{T}\right)+$ $c \cdot \mu_{2}=\pi\left(\mathbf{v}^{0}\right)+c^{\prime} \cdot \mu_{1}+c \cdot \mu_{2}$. Thus, $\pi$ is affine on $J$.

Proof (of Lemma 3.3). Case (i). Suppose $\{I, J\} \in \mathcal{E}_{0}$. Since $\pi, \pi^{1}, \pi^{2}$ are all continuous, we just prove that $\partial_{\mathbf{v}}$ is constant on $\operatorname{int}(J)$. If $\{I, J\} \in \mathcal{E}, \exists \mathbf{a} \in \frac{1}{q} \mathbb{Z}^{2}$ such that, setting $K=\{\mathbf{a}\} \in \mathcal{P}_{q, 0}$, one of the following two cases occurs.

Case 1. $(I, J, K) \in E\left(\pi, \mathcal{P}_{q}\right)$. Then $\pi_{I}(\mathbf{x})+\pi_{J}(\mathbf{y})=\pi_{K}(\mathbf{a})$ for all $\mathbf{x} \in I, \mathbf{y} \in$ $J, \mathbf{x} \oplus \mathbf{y}=\mathbf{a}$, or rewriting this, we have $\pi_{J}(\mathbf{x})=\pi_{K}(\mathbf{a})-\pi_{I}(\mathbf{a} \ominus \mathbf{x})$. For any $\mathbf{u} \in \operatorname{int}(J)$, it follows from Lemma 3.2 that $a \ominus \mathbf{u} \in \operatorname{int}(I)$. Since the right hand side is differentiable in the direction of $\mathbf{v}$ at $\mathbf{a} \ominus \mathbf{u}$, the left hand side is as well. The result in this case follows by the chain rule.

Case 2. $(I, K, J) \in E\left(\pi, \mathcal{P}_{q}\right)$. Then $\pi_{I}(\mathbf{x})+\pi_{K}(\mathbf{a})=\pi_{J}(\mathbf{y})$ for all $\mathbf{x} \in I, \mathbf{y} \in$ $J, \mathbf{x} \oplus \mathbf{a}=\mathbf{y}$, or rewriting this, we have $\pi_{J}(\mathbf{x} \oplus \mathbf{a})=\pi_{I}(\mathbf{x})+\pi_{K}(\mathbf{a})$. For any $\mathbf{u} \in \operatorname{int}(J)$, it follows from Lemma 3.2 that $\mathbf{u} \ominus \mathbf{a} \in \operatorname{int}(I)$. Since the right hand side is differentiable in the direction of $\mathbf{v}$, the left hand side is as well. The result again follows by the chain rule.

Case (ii). Suppose $\{I, J\} \in \mathcal{E} \backslash$. Using Lemma A.12 the proof follows similar to Case (i).

\section{A.9 Proof of Theorem 3.11}

Proof (of Theorem 3.11). Part (i). Suppose (1) does not have a unique solution. Let $\bar{\varphi}: \frac{1}{q} \mathbb{Z}^{2} \rightarrow \mathbb{R}$ be a non-trivial element in the kernel of the system above. Then for any $\epsilon,\left.\pi\right|_{\frac{1}{q} \mathbb{Z}^{2}}+\epsilon \bar{\varphi}$ also satisfies the system of equations. Let

$$
\epsilon=\min \left\{\Delta \pi_{F}(\mathbf{x}, \mathbf{y}) \neq 0 \mid F \in \Delta \mathcal{P}_{q},(\mathbf{x}, \mathbf{y}) \in \operatorname{vert}(F)\right\}
$$

Let $\bar{\pi}: \mathbb{R}^{2} \rightarrow \mathbb{R}$ be the continuous piecewise linear extension of $\varphi$ over $\mathcal{P}_{q}$ and set $\pi^{1}=\pi+\frac{\epsilon}{3\|\bar{\pi}\|_{\infty}} \bar{\pi}, \pi^{2}=\pi-\frac{\epsilon}{3\|\bar{\pi}\|_{\infty}} \bar{\pi}$. Note that $0<\|\bar{\pi}\|_{\infty}<\infty$ since $\bar{\varphi}$ comes from a non-trivial element in the kernel. We claim that $\pi^{1}, \pi^{2}$ are both minimal. As before, we show this for $\pi^{1}$, and the proof for $\pi^{2}$ is similar. Since $\left.\pi\right|_{\frac{1}{q} \mathbb{Z}^{2}}$ satisfies the system $(1)$ and $\bar{\varphi}$ is an element of the kernel, $\pi^{1}$ satisfies the system (1) as well. In particular, we have $\pi^{1}(\mathbf{0})=0, \pi^{1}(\mathbf{f})=1, \pi^{1}((0,1))=$ $0, \pi^{1}((0,1))=0, \pi^{1}((1,1))=0$.

Next, $\pi^{1}$ is symmetric because the symmetry conditions are implied here, that is, since we require that $\varphi(\mathbf{f})=1$, and since $\pi$ is minimal, $\Delta \pi(\mathbf{u}, \mathbf{f}-\mathbf{u})=0$ whenever $\mathbf{u} \in \frac{1}{q} \mathbb{Z}^{2}$, hence, by Theorem A.10, $\pi^{1}$ is symmetric. 
Lastly, we show that $\pi^{1}$ is subadditive. Let $\mathbf{u}, \mathbf{v} \in \frac{1}{q} \mathbb{Z}^{2}$. If $\Delta \pi(\mathbf{u}, \mathbf{v})=0$, then $\Delta \varphi(\mathbf{u}, \mathbf{v})=0$, as implied by the system of equations. Otherwise, if $\Delta \pi(\mathbf{u}, \mathbf{v})>0$, then

$$
\begin{aligned}
\Delta \pi^{1}(\mathbf{u}, \mathbf{v}) & =\Delta \pi(\mathbf{u}, \mathbf{v})+\frac{\epsilon}{3\|\bar{\varphi}\|_{\infty}} \bar{\varphi}(\mathbf{u})+\frac{\epsilon}{3\|\bar{\varphi}\|_{\infty}} \bar{\varphi}(\mathbf{v})-\frac{\epsilon}{3\|\bar{\varphi}\|_{\infty}} \bar{\varphi}(\mathbf{u} \oplus \mathbf{v}) \\
& \geq \Delta \pi_{F}(\mathbf{u}, \mathbf{v})-\frac{\epsilon}{3}-\frac{\epsilon}{3}-\frac{\epsilon}{3} \geq 0
\end{aligned}
$$

Therefore, by Theorem A.10 $\pi^{1}$ (and $\pi^{2}$ ) is subadditive and therefore minimal and valid. Therefore $\pi$ is not extreme.

Part (ii). Suppose there exist distinct, valid functions $\pi^{1}, \pi^{2}$ such that $\pi=$ $\frac{1}{2} \pi^{1}+\frac{1}{2} \pi^{2}$. Since $\pi$ is minimal and affine imposing in $\mathcal{P}_{q, 2}, \pi^{1}, \pi^{2}$ are minimal continuous piecewise linear functions over $\mathcal{P}_{q}$. Furthermore, $\left.\pi\right|_{\frac{1}{q} \mathbb{Z}^{2}}$ and, also $\left.\pi^{1}\right|_{\frac{1}{q} \mathbb{Z}^{2}},\left.\pi^{2}\right|_{\frac{1}{q} \mathbb{Z}^{2}}$ satisfy the system of equations (1). If this system has a unique solution, then $\pi=\pi^{1}=\pi^{2}$, which is a contradiction since $\pi^{1}, \pi^{2}$ were assumed distinct. Therefore $\pi$ is extreme.

On the other hand, if the system (1) does not have a unique solution, then by Theorem $3.11, \pi$ is not extreme. 